TIT/HEP-317

UCLA/96/TEP/11

hep-th/9603206

March, 1996

\title{
Gauge Symmetry Breaking through Soft Masses in Supersymmetric Gauge Theories
}

\author{
ERIC D'HOKER円 \\ Department of Physics and Astronomy, \\ University of California at Los Angeles \\ Los Angeles, CA 90024, USA \\ Yukihiro Mimurail and Norisuke Sakaif \\ Department of Physics, Tokyo Institute of Technology \\ Oh-okayama, Meguro, Tokyo 152, Japan
}

\begin{abstract}
We analyze the effects of soft supersymmetry breaking terms on $N=1$ supersymmetric QCD with $N_{f}$ flavors and color gauge group $S U\left(N_{c}\right)$. The mass squared of some squarks may be negative, as long as vacuum stability is ensured by a simple mass inequality. For $N_{f}<N_{c}$, we include the dynamics of the non-perturbative superpotential and use the original (s)quark and gauge fields, while for $N_{f}>N_{c}+1$, we formulate the dynamics in terms of dual (s)quarks and a dual gauge group $S U\left(N_{f}-N_{c}\right)$. The presence of negative squark mass squared terms leads to spontaneous breakdown of flavor and color symmetry. We determine this breaking pattern, derive the spectrum, and argue that the masses vary smoothly as one crosses from the Higgs phase into the confining phase.
\end{abstract}

\footnotetext{
* e-mail: dhoker@physics.ucla.edu

$\dagger$ e-mail: mim@th.phys.titech.ac.jp

$\ddagger$ e-mail: nsakai@th.phys.titech.ac.jp
} 


\section{Introduction}

The understanding of the dynamics of supersymmetric gauge theories has vastly improved through a combined use of the kinematical constraints of holomorphy and the dynamical assumption of duality. Whereas a number of exact results are known in theories with extended supersymmetry [1], a reliable qualitative picture has emerged in theories with simple $(N=1)$ supersymmetry as well [2] - [5]. Properties such as confinement and dynamical chiral symmetry breaking are found to interplay in a variety of different ways, depending on the numbers of colors $N_{c}$ and flavors $N_{f}$ of quarks and squarks.

Seiberg has proposed that these features are not restricted to $N=1$ supersymmetric gauge theories, but should survive - at least in a qualitative way - to nonsupersymmetric gauge theories. The models in which this proposal is perhaps most easily verified are those where supersymmetry is spontaneously broken (through the introduction of an additional sector of fields [6] - [7]) or those where soft, explicit supersymmetry breaking terms are added as a perturbation on the gauge dynamics [8]. The latter scheme of supersymmetry breaking was used in the original proposal of supersymmetric grand unified theories [9], [10] and provides a general framework for the usual formulation of the Minimal Supersymmetric Standard Model [11].

In a series of papers, it was argued that the addition of perturbative, soft supersymmetry breaking mass terms, with $m^{2} \geq 0$, essentially preserves the qualitative picture of the dynamics derived for $N=1$ supersymmetric QCD (SQCD) 12. An effective low energy theory is used in terms of color singlet meson and (for $N_{f} \geq N_{c}$ ) baryon fields, appropriate for the confining phase, and the effects of the non-perturbative superpotential of Affleck, Dine and Seiberg [3] are included. It was shown that flavor symmetry is dynamically broken from $S U\left(N_{f}\right) \times S U\left(N_{f}\right)$ down to $S U\left(N_{f}\right)$, just as in standard non-supersymmetric QCD. Indications are that, mostly, the dynamics admits a smooth transition to that of standard QCD, corresponding to the limit of large soft breaking mass terms. For special arrangements of $N_{c}$ and $N_{f}$, however, phase transitions may occur as the mass is increased, a possibility considered by [13].

In the present paper, we investigate $N=1$ supersymmetric QCD, again with soft supersymmetry breaking mass terms added, but this time with $m^{2}<0$ for at least some of the squark fields. For general values of the soft supersymmetry breaking mass terms with $m^{2}<0$, the Hamiltonian will become unbounded from below, thus 
destabilizing the entire theory. For certain simple ranges of the masses, however, we show that stable vacua exist through a balance between the soft supersymmetry breaking mass terms, the quartic $D^{2}$ term for the squark fields and ( for $N_{f}<N_{c}$ ) the non-perturbative effective potential. We argue that in these solutions, flavor as well as color symmetry are spontaneously broken through the vacuum expectation value of the squarks. We consider the most general soft supersymmetry breaking terms respecting $R$-symmetry, for simplicity.

For the analysis of spontaneous flavor and color symmetry breakdown when $N_{f}<N_{c}$, we formulate the dynamics in terms of the fundamental quark superfields instead of in terms of meson superfields, in contrast with the analysis in ref. 12. This choice appears more natural when dealing with the theory in the Higgs phase, rather than in the confining phase. In fact, the original calculation of the nonperturbative superpotential was justified precisely by considering gauge symmetry breakdown due to the vacuum expectation values of squark fields [3].

For small values of the soft supersymmetry breaking mass terms, we expect the confining and Higgs phases to be smoothly connected to one another, with matching low energy spectra. We find that this principle of complementarity between the confining and Higgs phases [14] can indeed be satisfied in these theories. To do so however, it appears to be necessary to consider the most general low energy effective action (consistent with internal symmetries and supersymmetry) in the confining phase. This action must include terms of higher order than was originally advocated in ref. 12.

For large values of the soft supersymmetry breaking mass terms, we expect the semi-classical spectrum for the Higgs phase, derived in terms of the fundamental quark superfields, to remain reliable. Thus, we shall calculate the semi-classical spectrum for this model for all ranges of soft breaking mass terms. For $N_{f}>N_{c}+1$, we will use the dual variables of ref. 迎, which are more appropriate to describe the spontaneous symmetry breakdown of flavor and (dual) color symmetry.

Finally, we note that there is a simple extension of standard SQCD, obtained by gauging also an additional anomaly-free $U(1)_{X}$ symmetry, and adding a FayetIlliopoulos $D$-term [6] for the corresponding gauge multiplet. (The simplest case would be where this $U(1)_{X}$ is just baryon number symmetry, but any anomaly-free $U(1)_{X}$ would do.) No explicit supersymmetry breaking terms are added; instead, a supersymmetric mass term is included in the superpotential, which stabilizes the vacuum. In this model, supersymmetry is broken spontaneously, and soft mass terms 
with $m^{2}<0$ automatically arise from the Fayet-Illiopoulos $D$-term. This model provides an economical realization of some of the effects of soft supersymmetry breaking mass terms generated directly by spontaneous breakdown of supersymmetry. It will be discussed in a companion paper. A different model has been analyzed which obtains the soft breaking terms from the spontaneous breakdown of supersymmetry [15.

The remainder of the paper is organized as follows. In Sect. 2, we analyze the case where $N_{f}<N_{c}$, and propose the use of the original squark fields as relevant effective degrees of freedom when some of the soft mass terms have $m^{2}<0$. Including kinetic terms, gauge couplings, soft breaking mass terms and the non-perturbative effective potential of ref.[3], we find the ranges of stability for the soft breaking mass terms, and the patterns of flavor and color symmetry breakdown. In Sect. 3, we analyze the case of supersymmetric QCD with $N_{f}>N_{c}+1$, with the help of the duality correspondence of Seiberg. Here, we add mass terms not to the original squark fields of the theory, but rather to the dual squark fields, which carry the dual color quantum numbers of $S U\left(N_{f}-N_{c}\right)$. In Sect. 4, we point out the difficulties encountered with our approach for $N_{f}=N_{c}$ and $N_{f}=N_{c}+1$ and discuss the region of validity of our analysis.

\section{Dynamics for $N_{f}<N_{c}$}

In this Section, we shall consider supersymmetric Yang-Mills theory with gauge group $S U\left(N_{c}\right)$ and $N_{f}$ flavors of squarks and quarks (with $N_{f}<N_{c}$ ), transforming under the representation $N_{c} \oplus \bar{N}_{c}$ of $S U\left(N_{c}\right)$. This theory is the natural supersymmetric extension of QCD, and will be referred to as SQCD. The corresponding chiral superfields

$$
\hat{Q}_{a}{ }^{i} \quad \hat{\bar{Q}}_{i}{ }^{a} \quad a=1, \cdots, N_{c} ; \quad i=1, \cdots, N_{f},
$$

contain the squark fields $Q$ and $\bar{Q}$ and the left-handed quark fields $\psi_{Q}$ and $\psi_{\bar{Q}}$ respectively. There is a natural color singlet meson chiral superfield $\hat{T}$, defined by

$$
\hat{T}_{i}^{j}=\hat{\bar{Q}}_{i}{ }^{a} \hat{Q}_{a}^{j}
$$

with scalar components $T_{i}^{j}$. Superfields are denoted by a cap on the scalar components. 
As a starting point we consider classical massless SQCD whose Lagrangian $\mathcal{L}_{0}$ is determined by $S U\left(N_{c}\right)$ gauge invariance, by requiring that the superpotential for the quark superfields vanish identically :

$$
\mathcal{L}_{0}=\int d^{4} \theta \operatorname{tr}\left\{\hat{Q}^{\dagger} e^{2 g \hat{V}} \hat{Q}+\hat{\bar{Q}} e^{-2 g \hat{V}} \hat{\bar{Q}}^{\dagger}\right\}+\frac{1}{2} \int d^{2} \theta \operatorname{tr} W W+\frac{1}{2} \int d^{2} \bar{\theta} \operatorname{tr} \bar{W} \bar{W}
$$

This theory has a global symmetry, $G_{f}=S U\left(N_{f}\right)_{Q} \times S U\left(N_{f}\right)_{\bar{Q}} \times U(1)_{B} \times U(1)_{R}$, under which the (bosonic or left-handed fermionic) component fields transform as

$$
\begin{array}{llll}
Q \rightarrow U_{c} Q U_{Q}^{\dagger} & & U_{Q} \in S U\left(N_{f}\right)_{Q} \\
\bar{Q} \rightarrow U_{\bar{Q}} \bar{Q} U_{c}^{\dagger} & & U_{\bar{Q}} \in S U\left(N_{f}\right)_{\bar{Q}} \\
T \rightarrow U_{\bar{Q}} T U_{Q}^{\dagger} & & U_{c} \in S U\left(N_{c}\right)
\end{array}
$$

with baryon number charges $1,-1$ and 0 respectively, and $R$-charges given by

$$
Q, \bar{Q}: 1-N_{c} / N_{f} \quad \psi_{Q}, \psi_{\bar{Q}}:-N_{c} / N_{f}
$$

A further classical axial baryon number $U(1)_{A B}$ symmetry suffers a color anomaly and is absent at the quantum level.

Exact nonperturbative results in supersymmetric gauge theories can be given for the $F$-type term which is a chiral superspace integral of a superpotential $W_{N P}$, 3] of the quark superfields $\hat{Q}$ and $\hat{\bar{Q}}$ given as follows

$$
\int d^{2} \theta W_{N P}(\hat{Q}, \hat{\bar{Q}})=\left(N_{c}-N_{f}\right) \Lambda^{3+2 N_{f} /\left(N_{c}-N_{f}\right)} \int d^{2} \theta(\operatorname{det} \hat{\bar{Q}} \hat{Q})^{-1 /\left(N_{c}-N_{f}\right)}
$$

The parameter $\Lambda$ has dimensions of mass and characterizes the strength of the nonperturbative effects. Henceforth, we shall set this parameter to $\Lambda=1$ to simplify notations; its dependence is easily recovered on dimensional grounds. Nonperturbative corrections are possible for $D$-type terms such as kinetic terms, but the exact results are not available since holomorphy places no direct restrictions on them «4.

\subsection{Soft Supersymmetry Breaking Mass Terms}

We choose to break supersymmetry explicitly, by adding to the Lagrangian $\mathcal{L}_{0}$ soft supersymmetry breaking terms for the quark supermultiplet. In general, soft breaking operators are defined as those which do not induce quadratic divergences to 
any order in perturbation theory (apart from vacuum diagrams). The only possible soft breaking operators can be summarized as in [8] : quadratic and cubic terms involving scalar fields with the same chirality; quadratic terms between scalar fields with opposite chirality (scalar masses); and gaugino masses. With the field contents of our model, the color gauge invariance forbids the cubic terms. The quadratic terms with the same chirality is forbidden if we want to maintain the flavor symmetry $S U\left(N_{f}\right)_{Q} \times S U\left(N_{f}\right)_{\bar{Q}}$. The gaugino masses violate the $U(1)_{R}$ symmetry. On the other hand squark masses that are separately equal on $Q$ and on $\bar{Q}$ will preserve the entire flavor group invariance $S U\left(N_{f}\right)_{Q} \times S U\left(N_{f}\right)_{\bar{Q}} \times U(1)_{B} \times U(1)_{R}$. Therefore we shall assume in this paper that squark masses are the only soft supersymmetry breaking terms.

Besides the above soft supersymmetry breaking operators, there are also the supersymmetry preserving masses for quark and antiquark superfields, which are evidently soft. However, their presence always explicitly breaks flavor symmetry. For example, if a common supersymmetric mass is given to all quarks, the flavor symmetry group $S U\left(N_{f}\right)_{Q} \times S U\left(N_{f}\right)_{\bar{Q}} \times U(1)_{B} \times U(1)_{R}$ will be broken down to $S U\left(N_{f}\right) \times U(1)_{B} \times U(1)_{R}$.

For the sake of simplicity, we shall add to the Lagrangian only soft supersymmetry breaking squark mass terms and neglect effects due to gaugino masses and supersymmetric flavor masses. Generic mass squared for squark and antisquarks are given by matrices $M_{Q}^{2}$ and $M_{\bar{Q}}^{2}$

$$
\mathcal{L}_{s b}=-\left\{\operatorname{tr} Q M_{Q}^{2} Q^{\dagger}+\operatorname{tr} \bar{Q}^{\dagger} M_{\bar{Q}}^{2} \bar{Q}\right\}
$$

As we remarked above, when $M_{Q}^{2}$ and $M_{\bar{Q}}^{2}$ are proportional to the identity matrix, the global flavor symmetry is unchanged : $S U\left(N_{f}\right)_{Q} \times S U\left(N_{f}\right)_{\bar{Q}} \times U(1)_{B} \times U(1)_{R}$.

The soft supersymmetry breaking masses can be expressed in terms of spurion superfields $\hat{\eta}$ and $\hat{\bar{\eta}}$ in superspace formalism [8]

$$
\begin{gathered}
\mathcal{L}_{s b}=-\int d^{4} \theta\left(\operatorname{tr} \hat{Q} \hat{\eta} \hat{Q}^{\dagger}+\operatorname{tr} \hat{\bar{Q}}^{\dagger} \hat{\bar{\eta}} \hat{\bar{Q}}\right) \\
\hat{\eta}=M_{Q}^{2} \theta^{2} \bar{\theta}^{2}, \quad \hat{\bar{\eta}}=M_{\bar{Q}}^{2} \theta^{2} \bar{\theta}^{2}
\end{gathered}
$$

This expression can be regarded as a spurion insertion to the kinetic term of the superfields $\hat{Q}$ and $\hat{\bar{Q}}$. As long as we insist on the spurion $\hat{\eta}$ and $\hat{\bar{\eta}}$ as the only source of supersymmetry breaking, we do not have any other possible supersymmetry breaking terms in the entire Lagrangian. This is because all the other terms including 
the nonperturbative superpotential (2.6) do not allow for any dependence on the spurion superfields $\hat{\eta}, \hat{\bar{\eta}}$, which are not chiral. If one assumes that chiral spurion superfields $\hat{\rho}=m \theta^{2}$ are also present as a source of supersymmetry breaking, one finds supersymmetry breaking terms of the F-type arising from the superpotential, such as the nonperturbative one (2.6). We will only use the spurion of the general superfields $\hat{\eta}, \hat{\bar{\eta}}$ in eq.(2.9) in this work.

When both $M_{Q}^{2}$ and $M_{\bar{Q}}^{2}$ are positive definite, the vacuum expectation value of the fields $Q$ and $\bar{Q}$ will vanish, and no spontaneous flavor symmetry breakdown is expected to occur (neglecting possible effects due to the nonperturbative potential in eq.(2.6) ). Color confinement is expected to take place and the physical spectrum should consist solely of color-singlet hadrons. This is the so-called confining phase. It is appropriate to reformulate the low energy dynamics of the theory in terms of color singlet fields only. This is precisely the approach followed by refs. [4], [12] which formulate the effective low energy dynamics of the theory in terms of the meson fields $T_{i}^{j}$, introduced in eq.(2.2).

When either $M_{Q}^{2}$ or $M_{\bar{Q}}^{2}$ is not positive definite, we expect the pattern of symmetry breaking to be substantially different. Global flavor symmetry should be spontaneously broken, and $Q$ and/or $\bar{Q}$ should acquire non-vanishing vacuum expectation values. These non-zero vacuum expectation values, in turn, are expected to break color $S U\left(N_{c}\right)$ and give mass to some of the gauge particles through the Higgs mechanism. This is the so-called Higgs phase. Exactly how much is left unbroken of the color symmetry depends upon the number of squark masses squared that are taken to be negative. We shall see that only a limited number of negative values can occur if the Hamiltonian is to remain bounded from below.

According to standard lore, (originally derived from lattice gauge theory) the confining and Higgs phases are smoothly connected to one another in at least some region of parameter space 14. There should be a one to one correspondence between the observables in both phases, suggesting that - in principle - color singlet meson fields could still be used to describe the dynamics of the Higgs phase. In practice, however, a formulation in terms of colored fields appears more suitable instead. Indeed, physical free quarks and certain massive gauge bosons are expected to appear in the low energy spectrum, and it is unclear how to represent these degrees of freedom in terms of meson variables. Thus, we shall use the original squark $Q, \bar{Q}$, quark $\psi_{Q}, \psi_{\bar{Q}}$, and gauge boson and fermion fields as physical variables at low

\footnotetext{
${ }^{*}$ For the case of $N_{f}<N_{c}$ under consideration, baryon fields do not appear.
} 
energy.

\subsection{Vacuum Stability}

We now have all the necessary ingredients to construct the potential of the squark fields : the effective non-perturbative contribution to the superpotential of (2.6), the soft supersymmetry breaking mass terms of (2.7) and finally, the square of the color $D$-term arising from the gauge couplings of the $\hat{\bar{Q}}$ and $\hat{Q}$ superfields, as given by (2.3). These contributions are easily worked out in terms of the squark fields, and we have ?

$$
\begin{aligned}
V= & \operatorname{tr}\left\{Q\left(Q^{\dagger} \bar{Q}^{\dagger} \bar{Q} Q\right)^{-1} Q^{\dagger}+\bar{Q}^{\dagger}\left(\bar{Q} Q Q^{\dagger} \bar{Q}^{\dagger}\right)^{-1} \bar{Q}\right\}|\operatorname{det} \bar{Q} Q|^{-2 /\left(N_{c}-N_{f}\right)} \\
& +\frac{g^{2}}{2}\left(\operatorname{tr} Q^{\dagger} t^{a} Q-\operatorname{tr} \bar{Q} t^{a} \bar{Q}^{\dagger}\right)^{2}+\left\{\operatorname{tr} Q M_{Q}^{2} Q^{\dagger}+\operatorname{tr} \bar{Q}^{\dagger} M_{\bar{Q}}^{2} \bar{Q}\right\}
\end{aligned}
$$

where the $S U\left(N_{c}\right)$ generators $t^{a}$ are normalized as $\operatorname{tr} t^{a} t^{b}=\frac{1}{2} \delta^{a b}$.

For zero soft supersymmetry breaking masses $M_{Q}^{2}$ and $M_{\bar{Q}}^{2}$, we recover standard massless SQCD. This theory possess no physical vacuum state, as can be seen by analyzing the full potential of (2.10) for $M_{Q}^{2}=M_{\bar{Q}}^{2}=0$. The potential diverges whenever an eigenvalue of $\bar{Q} Q$ tends to zero, thus driving its minimum away from $Q=0$ and $\bar{Q}=0$. Since both the quartic and the non-perturbative terms are positive, we obtain the absolute minimum configuration, with zero value of the potential, by setting $Q=\bar{Q}^{\dagger}$, (thus cancelling the quartic term) and letting $Q=$ $\bar{Q}^{\dagger} \rightarrow \infty$. This is a runaway solution and there is no physical vacuum state.

For positive values of squark mass squared, the above runaway solution is stabilized and a physical vacuum for SQCD is generated.

For generic matrices $M_{Q}^{2}$ and $M_{\bar{Q}}^{2}$, (in particular, with negative eigenvalues) the runaway directions can turn into directions along which the potential is unbounded from below. This is now possible, because the soft mass terms break supersymmetry explicitly, and the Hamiltonian need not be positive any longer. Restrictions on the soft mass terms guaranteeing that the potential be bounded from below can be obtained as follows. We use global flavor transformations to render the mass

*Notice that the normalization of the kinetic term, i.e. the Kähler potential in $\hat{Q}$ and $\hat{\bar{Q}}$ is canonically equal to 1 , so that no new normalization parameter need to be introduced here, in opposition to [12]. 
squared matrices $M_{Q}^{2}$ and $M_{\bar{Q}}^{2}$ diagonal in flavor, and denote their eigenvalues by $m_{Q_{i}}^{2}$ and $m_{\bar{Q}_{i}}^{2}$ respectively. Along the special directions $Q_{i}=\bar{Q}_{j}^{\dagger}$, the quartic potential vanishes identically and the non-perturbative potential tends to zero as $Q_{i}=\bar{Q}_{j}^{\dagger} \rightarrow$ $\infty$. If the potential is to be bounded from below, the remaining soft supersymmetry breaking mass terms in the potential must satisfy

$$
m_{Q_{i}}^{2}+m_{\bar{Q}_{j}}^{2} \geq 0
$$

for any pair of $i, j=1, \cdots N_{f}$. It is easy to see that this condition is also sufficient, as we shall establish later by explicit calculation of the potential in a convenient set of coordinates for the fields $Q$ and $\bar{Q}$.

Requiring in addition that the theory possess a well-defined ground state, leads to the condition above, but with strict inequalities, which is assumed henceforth.

The addition of supersymmetric quark mass terms would produce a stabilizing effect analogous to that of the positive soft mass squared terms. The pattern of gauge symmetry breakdown due to the supersymmetric mass terms has been analyzed in ref.[0]. If we add the supersymmetric mass terms, we can safely take the limit of vanishing soft mass terms without spoiling stability. Our results in this limit smoothly connect to the analysis in ref. [5].

\subsection{Description of Vacuum Configurations}

For simplicity, we shall explicitly analyze only the case where the mass squared for all $Q$ 's and $\bar{Q}$ 's are equal to $-m_{Q}^{2}$ and $m_{\bar{Q}}^{2}$ respectively, thus preserving the entire global symmetry $S U\left(N_{f}\right)_{Q} \times S U\left(N_{f}\right)_{\bar{Q}} \times U(1)_{B} \times U(1)_{R}$. The vacuum configuration is assumed to be Poincaré invariant and so that the values of $Q$ and $\bar{Q}$ in the vacuum are space-time independent. We shall determine these expectation values at the semi-classical level.

By making a global $S U\left(N_{c}\right) \times S U\left(N_{f}\right)_{Q} \times U(1)_{B}$ transformation on $Q$, and the remaining $S U\left(N_{f}\right)_{\bar{Q}} \times U(1)_{R}$ transformation on $\bar{Q}$, we can always rotate the vacuum expectation values of $Q$ and $\bar{Q}$ to the following arrangement

$$
\begin{aligned}
& Q=\left(\begin{array}{c}
Q_{(1)} \\
0
\end{array}\right) \quad\left(Q_{(1)}\right)_{i}{ }^{j}=Q_{i} \delta_{i}{ }^{j}
\end{aligned}
$$

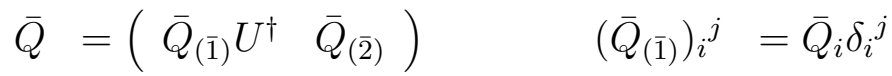


The eigenvalues $Q_{i}$ and $\bar{Q}_{i}, i=1, \cdots, N_{f}$, can be chosen to be real and positive. The matrix $U \in S U\left(N_{f}\right)$ arises from the fact that color must act on both $Q$ and $\bar{Q}^{\dagger}$ in the same way, so that independent color rotations can be made only on $Q$. The matrix $\bar{Q}_{(\overline{2})}$ is a general $N_{f} \times\left(N_{c}-N_{f}\right)$ complex matrix. This choice of variables is natural and has the advantage of considerably simplifying the non-perturbative potential contribution, especially the determinant.

To simplify the quartic contribution as well, it will be helpful to split up the generators $t^{p}$ of $S U\left(N_{c}\right)$ according to the splitting inherent in eq.(2.12). We separate $t^{p}$ into diagonal generators $H^{p}, p=1, \cdots N_{c}-1$, (of the Cartan subalgebra) and off diagonal (or root) generators $E^{p}$. The diagonal generators can be further separated as follows

$$
\begin{array}{ll}
H_{(0)}=\left(\begin{array}{cc}
I_{N_{f}} C_{1} & 0 \\
0 & -I_{N_{c}-N_{f}} C_{2}
\end{array}\right), & C_{1}=\sqrt{\left(N_{c}-N_{f}\right) / 2 N_{f} N_{c}} \\
H_{(1)}^{p}=\left(\begin{array}{cc}
h_{(1)}^{p} & 0 \\
0 & 0
\end{array}\right), & H_{(2)}^{q}=\left(\begin{array}{cc}
0 & 0 \\
0 & h_{(2)}^{q}
\end{array}\right) .
\end{array}
$$

Here, $I_{n}$ denotes the $n \times n$ identity matrix; the matrices $h_{(1)}^{p}$ and $h_{(2)}^{q}$ are of dimension $N_{f} \times N_{f}$ and $\left(N_{c}-N_{f}\right) \times\left(N_{c}-N_{f}\right)$ respectively, and are given by

$$
\left(h^{p}\right)_{i}{ }^{j}=\frac{\delta_{i}{ }^{j}}{\sqrt{2 p(p+1)}} \begin{cases}1 & \text { for } 1 \leq i \leq p \\ -p & \text { for } i=p+1 \\ 0 & \text { for } i \geq p+2\end{cases}
$$

for $h_{(1)}^{p}, p=1, \cdots, N_{f}-1$ and for $h_{(2)}^{p}, p=1, \cdots, N_{c}-N_{f}-1$. Explicit forms for the root generators $E^{a}$ will not be needed at this point.

The potential $V(Q, \bar{Q})$ in this basis becomes

$$
\begin{aligned}
& V=\left\{\operatorname{tr} Q_{(1)}^{-2}+\operatorname{tr} \bar{Q}_{(\overline{1})}^{-2}+\operatorname{tr} \bar{Q}_{(\overline{2})} \bar{Q}_{(\overline{2})}^{\dagger} \bar{Q}_{(\overline{1})}^{-1} U^{\dagger} Q_{(1)}^{-2} U \bar{Q}_{(\overline{1})}^{-1}\right\}\left[\prod_{i} Q_{i} \bar{Q}_{i}\right]^{-2 /\left(N_{c}-N_{f}\right)} \\
& +\frac{g^{2}}{2}\left\{\left(\operatorname{tr} Q_{(1)}^{2} h_{(1)}^{p}\right)^{2}+\left(\operatorname{tr} \bar{Q}_{(\overline{1})}^{2} h_{(1)}^{p}\right)^{2}-2\left(\operatorname{tr} Q_{(1)}^{2} h_{(1)}^{p}\right)\left(\operatorname{tr} U \bar{Q}_{(\overline{1})}^{2} U^{\dagger} h_{(1)}^{p}\right)\right. \\
& +\left(\operatorname{tr} \bar{Q}_{(\overline{2})}^{\dagger} \bar{Q}_{(\overline{2})} h_{(2)}^{q}\right)^{2}+\left[C_{1} \operatorname{tr}\left(Q_{(1)}^{2}-\bar{Q}_{(\overline{1})}^{2}\right)+C_{2} \operatorname{tr} \bar{Q}_{(\overline{2})}^{\dagger} \bar{Q}_{(\overline{2})}\right]^{2} \\
& +\left[\begin{array}{lll}
\operatorname{tr}\left(\bar{Q}_{(\overline{1})}\right. & \left.\bar{Q}_{(\overline{2})}\right)^{\dagger}\left(\bar{Q}_{(\overline{1})}\right. & \left.\left.\bar{Q}_{(\overline{2})}\right) E^{a}\right]^{2}
\end{array}\right\} \\
& -m_{Q}^{2} \operatorname{tr} Q_{(1)}^{2}+m_{\bar{Q}}^{2} \operatorname{tr} \bar{Q}_{(\overline{1})}^{2}+m_{\bar{Q}}^{2} \operatorname{tr} \bar{Q}_{(\overline{2})} \bar{Q}_{(\overline{2})}^{\dagger}
\end{aligned}
$$

We wish to minimize $V$ as a function of the variables $Q_{i}, \bar{Q}_{i}$, (i.e. the entries of $Q_{(1)}$ and $\bar{Q}_{(\overline{1})}$ respectively), $\bar{Q}_{(\overline{2})}$ and the $S U\left(N_{f}\right)$ matrix $U$. To do this, we shall show the following simple results. 
1. For fixed $Q_{i}, \bar{Q}_{i}$ and $U$, the minimum of $V$ in $\bar{Q}_{(\overline{2})}$ is at $\bar{Q}_{(\overline{2})}=0$;

2. For fixed $Q_{i}, \bar{Q}_{i}$ and $\bar{Q}_{(\overline{2})}=0$, the minimum as a function of $U$ is when $U \bar{Q}_{(\overline{1})} U^{\dagger}$ is diagonal, i.e. a permutation of the entries $\bar{Q}_{i}$ of $\bar{Q}_{(\overline{1})}$;

3. Under the conditions of 2., and fixed $Q_{0}^{2}=\sum_{i} Q_{i}^{2} / N_{f}$ and $\bar{Q}_{0}^{2}=\sum_{i} \bar{Q}_{i}^{2} / N_{f}$, the minimum of the potential is at $Q_{i}=Q_{0}$ and $\bar{Q}_{i}=\bar{Q}_{0}$, for all $i=1, \cdots, N_{f}$. (We have assumed, without loss of generality, that $Q_{0} \geq 0, \bar{Q}_{0} \geq 0$.)

4. The remaining potential is of the same form as the one for $N_{f}=1$, and always admits a non-trivial solution with $Q_{0} \neq 0$ and $\bar{Q}_{0} \neq 0$.

To show 1., we notice that every term separately involving $\bar{Q}_{(\overline{2})}$ in $V$, assumes its absolute minimum at $\bar{Q}_{(\overline{2})}=0$. The only term for which this is not a priori obvious is $\left[C_{1} \operatorname{tr}\left(Q_{(1)}^{2}-\bar{Q}_{(\overline{1})}^{2}\right)+C_{2} \operatorname{tr} \bar{Q}_{(\overline{2})}^{\dagger} \bar{Q}_{(\overline{2})}\right]^{2}$. We make use of the fact that with the signassignment of the mass terms, $m_{Q}^{2}>0$ and $m_{\bar{Q}}^{2}>0$, we must have $\operatorname{tr} Q_{(1)}^{2}>\operatorname{tr} \bar{Q}_{(\overline{1})}^{2}$. Since $C_{1}>0$ and $C_{2}>0$, it follows that the above term also achieves its absolute minimum when $\bar{Q}_{(\overline{2})}=0$.

To show 2., we set $\bar{Q}_{(\overline{2})}=0$, and notice that only a single term depends upon the unitary matrix $U$, given by $\left(\operatorname{tr} Q_{(1)}^{2} h_{(1)}^{p}\right)\left(\operatorname{tr} U \bar{Q}_{(\overline{1})}^{2} U^{\dagger} h_{(1)}^{p}\right)$. Varying with respect to $U$ to find the extrema, we have

$$
\left[h_{(1)}^{p}, U \bar{Q}_{(\overline{1})}^{2} U^{\dagger}\right] \operatorname{tr} Q_{(1)}^{2} h_{(1)}^{p}=0
$$

Assuming that $Q_{(1)}$ takes on generic values, this equation requires that $U \bar{Q}_{(\overline{1})}^{2} U^{\dagger}$ should commute with all $h_{(1)}^{p}$ matrices, i.e. with all diagonal matrices. This is possible only when $U \bar{Q}_{(\overline{1})}^{2} U^{\dagger}$ itself is diagonal. But then the new matrix will be given by the matrix $\bar{Q}_{(\overline{1})}$ in which the entries have been interchanged by a permutation given by $U$. We shall denote this permutation by $\sigma_{U}$ or just $\sigma$ for short, and its action on $\bar{Q}_{i}$ is then conveniently represented by $\bar{Q}_{i} \rightarrow \bar{Q}_{\sigma(i)}$. To find the absolute minimum amongst the $\left(N_{f}\right)$ ! permutations, we make use of the following inner product formulas for the matrices $h$, and any two diagonal matrices $A$ and $B$, with entries $A_{i}$ and $B_{i}$ respectively :

$$
\sum_{p=1}^{N-1} \operatorname{tr}\left(A h^{p}\right) \operatorname{tr}\left(B h^{p}\right)=\frac{1}{2 N} \sum_{i<j}\left(A_{i}-A_{j}\right)\left(B_{i}-B_{j}\right)
$$

As a result, the value of the term involving $U$ is re-expressed as

$$
-g^{2}\left(\operatorname{tr} Q_{(1)}^{2} h_{(1)}^{p}\right)\left(\operatorname{tr} U \bar{Q}_{(\overline{1})}^{2} U^{\dagger} h_{(1)}^{p}\right)=-\frac{g^{2}}{2 N_{f}} \sum_{i<j}\left(Q_{i}^{2}-Q_{j}^{2}\right)\left(\bar{Q}_{\sigma(i)}^{2}-\bar{Q}_{\sigma(j)}^{2}\right)
$$


It is easy to show that the absolute minimum of this term occurs when all terms in the sum are separately positive. This happens when the orderings of $Q_{i}$ and $\bar{Q}_{i}$ are correlated as follows:

$$
\begin{gathered}
Q_{i_{1}}^{2} \geq Q_{i_{2}}^{2} \geq Q_{i_{3}}^{2} \geq \cdots \geq Q_{i_{N_{f}}}^{2} \\
\bar{Q}_{\sigma\left(i_{1}\right)}^{2} \geq \bar{Q}_{\sigma\left(i_{2}\right)}^{2} \geq \bar{Q}_{\sigma\left(i_{3}\right)}^{2} \geq \cdots \geq \bar{Q}_{\sigma\left(i_{N_{f}}\right)}^{2}
\end{gathered}
$$

This completes the argument for point 2.

To show 3., we use (2.17) to express all inner products of traces; then, under the conditions of 1 . and 2 . we have

$$
\begin{aligned}
V= & \sum_{i}\left(Q_{i}^{-2}+\bar{Q}_{i}^{-2}\right)\left[\prod_{j} Q_{j} \bar{Q}_{j}\right]^{-2 /\left(N_{c}-N_{f}\right)} \\
& +\frac{g^{2}}{4 N_{f}} \sum_{i<j}\left\{\left(Q_{i}^{2}-Q_{j}^{2}\right)^{2}+\left(\bar{Q}_{i}^{2}-\bar{Q}_{j}^{2}\right)^{2}-2\left(Q_{i}^{2}-Q_{j}^{2}\right)\left(\bar{Q}_{\sigma(i)}^{2}-\bar{Q}_{\sigma(j)}^{2}\right)\right\} \\
& +g^{2} \frac{N_{c}-N_{f}}{4 N_{c} N_{f}}\left\{\sum_{i} Q_{i}^{2}-\sum_{i} \bar{Q}_{i}^{2}\right\}^{2}-m_{Q}^{2} \sum_{i} Q_{i}^{2}+m_{\bar{Q}}^{2} \sum_{i} \bar{Q}_{i}^{2}
\end{aligned}
$$

Keeping $Q_{0}^{2}=\sum_{i} Q_{i}^{2} / N_{f}$, and $\bar{Q}_{0}^{2}=\sum_{i} \bar{Q}_{i}^{2} / N_{f}$ fixed, while varying $Q_{i}$ and $\bar{Q}_{i}$ the terms on the third line in eq.(2.20) are fixed under this variation. It is easy to see that the non-perturbative term on the first line of eq.(2.20) assumes its absolute minimum when $Q_{i}=Q_{0}$ and $\bar{Q}_{i}=\bar{Q}_{0}$ for all $i=1, \cdots, N_{f}$. (Recall that we arranged all $Q_{i}$ and $\bar{Q}_{i}$ to be positive.) But, for these values, the terms on the second line automatically assume their absolute minimum value : 0 . Thus, the configuration where $Q_{i}=Q_{0}$ and $\bar{Q}_{i}=\bar{Q}_{0}$ for all $i=1, \cdots, N_{f}$, gives the absolute minimum for the entire potential $V$ subject to the constraint $Q_{0}^{2}=\sum_{i} Q_{i}^{2} / N_{f}$, and $\bar{Q}_{0}^{2}=\sum_{i} \bar{Q}_{i}^{2} / N_{f}$, which proves point 3 .

To show 4., we substitute the values $Q_{i}=Q_{0}$ and $\bar{Q}_{i}=\bar{Q}_{0}$ for all $i=1, \cdots, N_{f}$ into the expression for $V$ and obtain

$$
V / N_{f}=\left(Q_{0}^{2}+\bar{Q}_{0}^{2}\right)\left[Q_{0} \bar{Q}_{0}\right]^{-2 \gamma}+\frac{g^{2}}{4 \gamma}\left(Q_{0}^{2}-\bar{Q}_{0}^{2}\right)^{2}-m_{Q}^{2} Q_{0}^{2}+m_{\bar{Q}}^{2} \bar{Q}_{0}^{2}
$$

We have introduced a constant that contains all the remaining dependence on $N_{c}$ and $N_{f}$ :

$$
\gamma=\frac{N_{c}}{N_{c}-N_{f}}>1
$$


Clearly, the reduced potential assumes the same form as for a single flavor, but with modified value of $\gamma$. The minimum conditions are

$$
\begin{aligned}
& 0=(\gamma-1) Q_{0}^{-2 \gamma} \bar{Q}_{0}^{-2 \gamma}+\gamma Q_{0}^{-2-2 \gamma} \bar{Q}_{0}^{2-2 \gamma}-\frac{g^{2}}{2 \gamma}\left(Q_{0}^{2}-\bar{Q}_{0}^{2}\right)+m_{Q}^{2} \\
& 0=(\gamma-1) Q_{0}^{-2 \gamma} \bar{Q}_{0}^{-2 \gamma}+\gamma Q_{0}^{2-2 \gamma} \bar{Q}_{0}^{-2-2 \gamma}+\frac{g^{2}}{2 \gamma}\left(Q_{0}^{2}-\bar{Q}_{0}^{2}\right)-m_{\bar{Q}}^{2}
\end{aligned}
$$

It is very convenient to introduce new variables $v$ and $x$ with $0 \leq x \leq 1$, in terms of which we have

$$
Q_{0}^{2}=v^{2}(1+x) \quad \bar{Q}_{0}^{2}=v^{2}(1-x),
$$

The equations for $Q_{0}$ and $\bar{Q}_{0}$ are equivalent to requiring $v^{2}=v_{1}^{2}(x)=v_{2}^{2}(x)$, where the functions $v_{1}^{2}(x)$ and $v_{2}^{2}(x)$ are given by

$$
\begin{aligned}
v_{1}^{2}(x) & =\left(m_{\bar{Q}}^{2}-m_{Q}^{2}\right)^{-1 / 2 \gamma}\left(1-x^{2}\right)^{-1 / 2-1 / 2 \gamma}\left\{4 \gamma-2+2 x^{2}\right\}^{1 / 2 \gamma} \\
v_{2}^{2}(x) & =\frac{\gamma}{g^{2}}\left\{\frac{m_{Q}^{2}+m_{\bar{Q}}^{2}}{2 x}-\frac{\gamma\left(m_{\bar{Q}}^{2}-m_{Q}^{2}\right)}{2 \gamma-1+x^{2}}\right\}
\end{aligned}
$$

As $x$ ranges from 0 to 1 , the function $v_{1}^{2}(x)$ is monotonically increasing to $\infty$ as $x$ approaches 1 , while $v_{2}^{2}(x)$ is monotonically decreasing from $\infty$ as $x$ approaches 0 . From this behavior, it is clear that (2.25) admits a unique solution for any arrangement of $g^{2}>0, \gamma>1$ and masses $m_{\bar{Q}}^{2}-m_{Q}^{2}>0$. (Henceforth, we find it convenient to express physical parameters, such as masses of various particles, in terms of the original dimensionless parameters $\gamma$ and $g$, and the quantities $v$ and $x$ determined by the implicit equations of (2.23).)

It is easy to find an approximate solution for the limit where both $Q_{0}$ and $\bar{Q}_{0}$ are large. This limit corresponds to $v^{2}$ large, and according to (2.25) this means $x$ small :

$$
\begin{aligned}
v^{2} & \sim(4 \gamma-2)^{1 / 2 \gamma}\left(m_{\bar{Q}}^{2}-m_{Q}^{2}\right)^{-1 / 2 \gamma} \\
x & \sim \frac{\gamma}{2 g^{2}}(4 \gamma-2)^{-1 / 2 \gamma}\left(m_{\bar{Q}}^{2}+m_{Q}^{2}\right)\left(m_{\bar{Q}}^{2}-m_{Q}^{2}\right)^{1 / 2 \gamma}
\end{aligned}
$$

This situation will occur if the masses $m_{Q}$ and $m_{\bar{Q}}$ are small compared with the non-perturbative scale $\Lambda$, or when these masses are large, but only their difference is small compared to $\Lambda$.

If we let the mass squared for quarks to increase from negative values $-m_{Q}^{2}$ to positive values, we find that our solutions smoothly connect to the positive mass 
squared case in ref. [12]. Their analysis was done using meson variables appropriate for the confining phase, whereas ours uses the colored fundamental quark variables appropriate for the Higgs phase. This smooth transition gives further evidence for the complementarity of confining and Higgs pictures in the case of quarks in the fundamental representation [14]. We shall further substantiate this complementarity by comparing the mass spectra in Section 2.6.

\subsection{Spectrum and Unbroken Symmetries}

It was shown previously that soft supersymmetry breaking squark mass terms may be introduced, which respect the full $S U\left(N_{c}\right) \times S U\left(N_{f}\right)_{Q} \times S U\left(N_{f}\right)_{\bar{Q}} \times U(1)_{B} \times U(1)_{R}$ symmetry. Under the assumption that $m_{Q}^{2}<m_{\bar{Q}}^{2}$, we found that the squark fields acquire non-zero vacuum expectation values,

$$
\langle 0|Q| 0\rangle=\left(\begin{array}{c}
Q_{0} I_{N_{f}} \\
0
\end{array}\right) \quad\langle 0|\bar{Q}| 0\rangle=\left(\begin{array}{cc}
\bar{Q}_{0} I_{N_{f}} & 0
\end{array}\right)
$$

with $Q_{0}$ and $\bar{Q}_{0}$ given by eqs.(2.24) and (2.25). In view of these expectation values, color and flavor symmetries are spontaneously broken to

$$
S U\left(N_{c}-N_{f}\right) \times S U\left(N_{f}\right)_{V} \times U(1)_{B}
$$

Here, $S U\left(N_{f}\right)_{V}$ is the diagonal subgroup of the $S U\left(N_{f}\right)$ subgroup of color and the flavor group $S U\left(N_{f}\right)_{Q} \times S U\left(N_{f}\right)_{\bar{Q}}$. The spectrum of the model will transform under linear representations of this unbroken group.

In this Section, we shall calculate the masses of the various elementary constituents, in terms of the breaking parameters evaluated previously. We begin by separating the field variables according to irreducible representations of $S U\left(N_{c}-\right.$ $\left.N_{f}\right) \times S U\left(N_{f}\right)_{V}$. Different fields can mix only (and will mix in fact) if they have the same spin and transform under the same irreducible representation of $S U\left(N_{c}-\right.$ $\left.N_{f}\right) \times S U\left(N_{f}\right)_{V}$. Thus, the mass matrix can be diagonalized in each respective representation subspace. The separation of variables is effected as follows

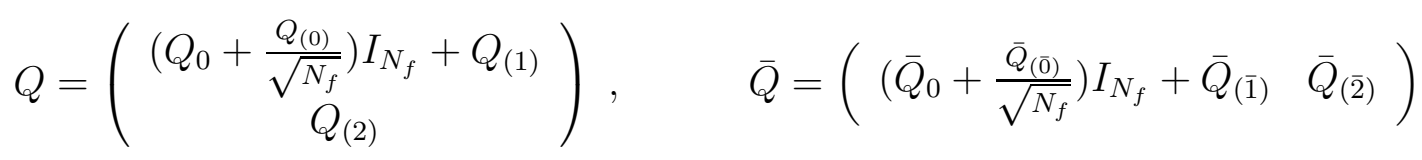




$$
\begin{aligned}
& \psi_{Q}=\left(\begin{array}{c}
\frac{\psi_{(0)}}{\sqrt{N_{f}}} I_{N_{f}}+\psi_{(1)} \\
\psi_{(2)}
\end{array}\right), \quad \psi_{\bar{Q}}=\left(\begin{array}{ll}
\frac{\psi_{(\overline{0})}}{\sqrt{N_{f}}} I_{N_{f}}+\psi_{(\overline{1})} & \psi_{(\overline{2})}
\end{array}\right) \\
& A_{\mu}=\left(\begin{array}{cc}
A_{\mu(1)} & A_{\mu(2)} \\
A_{\mu(2)}^{\dagger} & A_{\mu(3)}
\end{array}\right)+A_{\mu(0)} H_{(0)}, \quad \lambda=\left(\begin{array}{cc}
\lambda_{(1)} & \lambda_{(2)} \\
\lambda_{(\overline{2})} & \lambda_{(3)}
\end{array}\right)+\lambda_{(0)} H_{(0)},
\end{aligned}
$$

The fields $Q_{(0)}, \bar{Q}_{(\overline{0})}, \psi_{(0)}, \psi_{(\overline{0})}, A_{\mu(0)}$ and $\lambda_{(0)}$ are uniquely defined, provided we insist on the following conditions

$$
\begin{aligned}
& 0=\operatorname{tr} Q_{(1)}=\operatorname{tr} \bar{Q}_{(\overline{1})}=\operatorname{tr} \psi_{(1)}=\operatorname{tr} \psi_{(\overline{1})} \\
& 0=\operatorname{tr} A_{\mu(1)}=\operatorname{tr} A_{\mu(3)}=\operatorname{tr} \lambda_{(1)}=\operatorname{tr} \lambda_{(3)}
\end{aligned}
$$

The transformation properties and multiplicity of these fields are summarized in the tables 1 - 3 for fields of spin 1 , spin $1 / 2$ and spin 0 respectively.

\subsubsection{Spin 1 Masses}

Gauge boson mass terms are read off from the $D$-term part of the Lagrangian density in eq.(2.3), and are given by

$$
-g^{2} A_{\mu}^{p} A^{\mu q} \operatorname{tr}\left\{\left\langle 0\left|Q^{\dagger}\right| 0\right\rangle t^{p} t^{q}\langle 0|Q| 0\rangle+\langle 0|\bar{Q}| 0\rangle t^{p} t^{q}\left\langle 0\left|\bar{Q}^{\dagger}\right| 0\right\rangle\right\}
$$

It is straightforward to read off the masses of the gauge bosons. The would-beGoldstone bosons that are eaten in order to give these vector bosons mass are identified by inspecting the linear coupling of the scalar fields to the gauge bosons. These couplings are given by

$$
\begin{aligned}
i g & \left\{\frac{1}{\sqrt{\gamma}} A_{\mu(0)} \partial^{\mu}\left(Q_{0} Q_{(0)}+\bar{Q}_{0} \bar{Q}_{(\overline{0})}^{*}\right)+\operatorname{tr} A_{\mu(1)} \partial^{\mu}\left(Q_{0} Q_{(1)}+\bar{Q}_{0} \bar{Q}_{(\overline{1})}^{\dagger}\right)\right. \\
& \left.+\operatorname{tr} A_{\mu(2)} \partial^{\mu}\left(Q_{0} Q_{(2)}+\bar{Q}_{0} \bar{Q}_{(\overline{2})}^{\dagger}\right)\right\}+ \text { c.c. }
\end{aligned}
$$

The properties of the vector bosons are summarized in the table 11.

\subsubsection{Spin 1/2 Masses}

The fermion masses are governed by contributions both from the superpotential (contribution $V_{1}$ ) and from the mixing of the quarks and the gauginos in the $D$ term (contribution $V_{2}$ ) in eq.(2.3). The first contribution, in terms of the irreducible 


\begin{tabular}{|c|c|c|c|}
\hline Spin 1 & Masses & Multiplicity & $S U\left(N_{f}\right) \times S U\left(N_{c}-N_{f}\right)$ \\
\hline \hline$A_{\mu(0)}$ & $g v \sqrt{2 / \gamma}$ & 1 & $1 \otimes 1$ \\
\hline$A_{\mu(1)}$ & $g v \sqrt{2}$ & $N_{f}^{2}-1$ & adjoint $\otimes 1$ \\
\hline$A_{\mu(2)}$ & $g v$ & $2 N_{f}\left(N_{c}-N_{f}\right)$ & $N_{f}^{*} \otimes\left(N_{c}-N_{f}\right) \oplus N_{f} \otimes\left(N_{c}-N_{f}\right)^{*}$ \\
\hline$A_{\mu(3)}$ & 0 & $\left(N_{c}-N_{f}\right)^{2}-1$ & $1 \otimes$ adjoint \\
\hline
\end{tabular}

Table 1: The spin 1 fields and their masses $\left(N_{f}<N_{c}\right)$

fields of eq.(2.29) is given by

$$
\begin{aligned}
V_{1}= & \frac{1}{2} \gamma M \psi_{(0)} \psi_{(0)}+\frac{1}{2} \gamma \bar{M} \psi_{(\overline{0})} \psi_{(\overline{0})}+(\gamma-1) \sqrt{M \bar{M}} \psi_{(0)} \psi_{(\overline{0})} \\
& +\frac{1}{2} M \operatorname{tr} \psi_{(1)} \psi_{(1)}+\frac{1}{2} \bar{M} \operatorname{tr} \psi_{(\overline{1})} \psi_{(\overline{1})}+\sqrt{M \bar{M}} \operatorname{tr} \psi_{(\overline{2})} \psi_{(2)}+\text { c.c. }
\end{aligned}
$$

Here, we have introduced the notation $M$ and $\bar{M}$ for the effective masses coming from the non-perturbative potential. They can be expressed as follows in terms of the vacuum expectation values $Q_{0}, \bar{Q}_{0}$ or in terms of the basic parameters :

$$
\begin{aligned}
& M=Q_{0}^{-\gamma-1} \bar{Q}_{0}^{-\gamma+1}=(1-x)\left(m_{\bar{Q}}^{2}-m_{Q}^{2}\right)^{1 / 2}\left(4 \gamma-2+2 x^{2}\right)^{-1 / 2} \\
& \bar{M}=Q_{0}^{-\gamma+1} \bar{Q}_{0}^{-\gamma-1}=(1+x)\left(m_{\bar{Q}}^{2}-m_{Q}^{2}\right)^{1 / 2}\left(4 \gamma-2+2 x^{2}\right)^{-1 / 2}
\end{aligned}
$$

Similarly, the contribution $V_{2}$ may be decomposed in terms of the irreducible fields of eq.(2.29), and we obtain

$$
\begin{aligned}
V_{2}= & -i g / \sqrt{\gamma} Q_{0} \lambda_{(0)} \psi_{(0)}+i g / \sqrt{\gamma} \bar{Q}_{0} \lambda_{(0)} \psi_{(\overline{0})} \\
& -i \sqrt{2} g Q_{0}\left(\operatorname{tr} \lambda_{(1)} \psi_{(1)}+\operatorname{tr} \lambda_{(2)} \psi_{(2)}\right) \\
& +i \sqrt{2} g \bar{Q}_{0}\left(\operatorname{tr} \lambda_{(1)} \psi_{(\overline{1})}+\operatorname{tr} \lambda_{(\overline{2})} \psi_{(\overline{2})}\right)+\text { c.c. }
\end{aligned}
$$

From these mass terms, it is clear that the following groups of fields mix amongst each other, but do not mix from one group to the next. Singlets under $S U\left(N_{f}\right)$ : $\left(\psi_{(0)}, \psi_{(\overline{0})}, \lambda_{(0)}\right)$; adjoint under $S U\left(N_{f}\right):\left(\psi_{(1)}, \psi_{(\overline{1})}, \lambda_{(1)}\right)$; fundamental under $S U\left(N_{f}\right):\left(\psi_{(2)}, \psi_{(\overline{2})}, \lambda_{(2)}, \lambda_{(\overline{2})}\right)$. Thus, the mass matrix may be diagonalized in each set separately.

In the set $\left(\psi_{(0)}, \psi_{(\overline{0})}, \lambda_{(0)}\right)$, the masses are governed by the cubic equation

$$
\begin{aligned}
0= & \mathcal{M}^{3}-\mathcal{M}^{2} \gamma(M+\bar{M})+\mathcal{M}\left\{(2 \gamma-1) M \bar{M}+2 \frac{g^{2}}{\gamma} v^{2}\right\} \\
& -g^{2}\left(\bar{Q}_{0} \sqrt{M}+Q_{0} \sqrt{\bar{M}}\right)^{2}+2 \frac{g^{2}}{\gamma} Q_{0} \bar{Q}_{0} \sqrt{M \bar{M}}
\end{aligned}
$$


It is instructive to evaluate the solutions in the limit where $M, \bar{M} \ll g v$, and we find the masses

$$
\begin{aligned}
\mathcal{M}_{(0)}^{0} & =\frac{\gamma}{2 v^{2}}\left(\bar{Q}_{0} \sqrt{M}+Q_{0} \sqrt{\bar{M}}\right)^{2}-\frac{1}{v^{2}} Q_{0} \bar{Q}_{0} \sqrt{M \bar{M}}+\mathcal{O}\left(M^{2}\right) \\
\mathcal{M}_{(0)}^{ \pm} & = \pm \frac{i \sqrt{2}}{\sqrt{\gamma}} g v+\frac{\gamma}{2}(M+\bar{M})-\frac{1}{2} \mathcal{M}_{(0)}^{0}+\mathcal{O}\left(M^{2}\right)
\end{aligned}
$$

The field $\psi_{(0)}^{0}$, with mass $\mathcal{M}_{(0)}^{0}$, that is light in this limit, is given approximately by

$$
\psi_{(0)}^{0}=\frac{1}{\sqrt{2} v}\left(\bar{Q}_{0} \psi_{(0)}+Q_{0} \psi_{(\overline{0})}\right)
$$

while the other two heavy fields correspond to the orthogonal linear combinations.

In the set $\left(\psi_{(1)}, \psi_{(\overline{1})}, \lambda_{(1)}\right)$, the masses of the three irreducible representations again satisfy a cubic equation, given by

$$
0=\mathcal{M}^{3}-\mathcal{M}^{2}(M+\bar{M})+\mathcal{M} M \bar{M}+4 g^{2} v^{2} \mathcal{M}-2 g^{2}\left(\bar{Q}_{0}^{2} M+Q_{0}^{2} \bar{M}\right)
$$

In the limit where $M, \bar{M} \ll g v$, we find the masses

$$
\begin{aligned}
\mathcal{M}_{(1)}^{0} & =\frac{1}{2 v^{2}}\left(\bar{Q}_{0}^{2} M+Q_{0}^{2} \bar{M}\right)+\mathcal{O}\left(M^{2}\right) \\
\mathcal{M}_{(1)}^{ \pm} & = \pm 2 i g v+\frac{1}{2}(M+\bar{M})-\frac{1}{2} \mathcal{M}_{(1)}^{0}+\mathcal{O}\left(M^{2}\right)
\end{aligned}
$$

The field $\psi_{(1)}^{0}$ of mass $\mathcal{M}_{(1)}^{0}$, that is light in this limit is given approximately by

$$
\psi_{(1)}^{0}=\frac{1}{\sqrt{2} v}\left(\bar{Q}_{0} \psi_{(1)}+Q_{0} \psi_{(\overline{1})}\right)
$$

In the set $\left(\psi_{(2)}, \psi_{(\overline{2})}, \lambda_{(2)}, \lambda_{(\overline{2})}\right)$, the masses of the four irreducible representations satisfy a quartic equation which is even, given by

$$
0=\mathcal{M}^{4}+\mathcal{M}^{2}\left(4 g^{2} v^{2}-M \bar{M}\right)+4 g^{4} Q_{0}^{2} \bar{Q}_{0}^{2}
$$

In the limit where $M, \bar{M} \ll g v$, we find

$$
\begin{aligned}
& \pm \mathcal{M}_{(2)}= \pm i \sqrt{2} g Q_{0}^{2}+\mathcal{O}\left(M^{2}\right) \\
& \pm \overline{\mathcal{M}}_{(2)}= \pm i \sqrt{2} g \bar{Q}_{0}^{2}+\mathcal{O}\left(M^{2}\right)
\end{aligned}
$$

From the mass eigenvalue equations for fermions, it is evident that $\mathcal{M}=0$ eigenvalues can never occur so that all fermions are always massive.

A summary of all spin 1/2 fields and masses is given in the table 2. 


\begin{tabular}{|c|c|c|c|}
\hline Spin 1/2 & Masses & Multiplicity & $S U\left(N_{f}\right) \times S U\left(N_{c}-N_{f}\right)$ \\
\hline \hline$\psi_{(0)}, \psi_{(\overline{0})}, \lambda_{(0)}$ & $\mathcal{M}_{(0)}^{ \pm}, \mathcal{M}_{(0)}^{0}$ & 3 & $1 \otimes 1$ \\
\hline$\psi_{(1)}, \psi_{(\overline{1})}, \lambda_{(1)}$ & $\mathcal{M}_{(1)}^{ \pm}, \mathcal{M}_{(1)}^{0}$ & $3\left(N_{f}^{2}-1\right)$ & adjoint $\otimes 1$ \\
\hline$\psi_{(2)}, \lambda_{(2)}$, & $\pm \mathcal{M}_{(2)}$ & $2 N_{f}\left(N_{c}-N_{f}\right)$ & $2 N_{f}^{*} \otimes\left(N_{c}-N_{f}\right)$ \\
$\psi_{(\overline{2})}, \lambda_{(\overline{2})}$ & $\pm \mathcal{M}_{(2)}$ & $2 N_{f}\left(N_{c}-N_{f}\right)$ & $2 N_{f} \otimes\left(N_{c}-N_{f}\right)^{*}$ \\
\hline
\end{tabular}

Table 2: The spin $1 / 2$ fields and their masses $\left(N_{f}<N_{c}\right)$

\subsubsection{Spin 0 Masses}

Finally, the masses of spin 0 bosons are again found by decoupling the equations according to the fields of eq.(2.29). There are three groups of irreducible representations : the singlets under $S U\left(N_{f}\right):\left(Q_{(0)}, \bar{Q}_{(\overline{0})}\right)$, the adjoints under $S U\left(N_{f}\right)$ : $\left(Q_{(1)}, \bar{Q}_{(\overline{1})}\right)$ and the fundamental under $S U\left(N_{f}\right):\left(Q_{(2)}, \bar{Q}_{(\overline{2})}\right)$. The dynamics of the scalar fields is governed by the potential $V$ of eq.(2.10), and we shall now analyze the mass spectrum for each of the irreducible groups in turn.

The dynamics for the group $\left(Q_{(0)}, \bar{Q}_{(\overline{0})}\right)$ may be split as follows. First, the phases of $Q_{(0)}$ and $\bar{Q}_{(\overline{0})}$ are massless. One of them is eaten by the massive gauge field $A_{\mu(0)}$, while the other is a true Goldstone boson, and has zero mass. Second, the masses of the norms of these fields is given by the reduced potential of (2.21), and thus characterized by a quadratic equation

$$
\begin{gathered}
0=\left(\mathcal{M}^{2}-2 \gamma(\gamma-1) M \bar{M}-2 \gamma(\gamma+1) M^{2}-\frac{g^{2}}{\gamma} Q_{0}^{2}\right) \times \\
\left(\mathcal{M}^{2}-2 \gamma(\gamma-1) M \bar{M}-2 \gamma(\gamma+1) \bar{M}^{2}-\frac{g^{2}}{\gamma} \bar{Q}_{0}^{2}\right) \\
-\left(4 \gamma(\gamma-1) \frac{v^{2}}{Q_{0} \bar{Q}_{0}} M \bar{M}-\frac{g^{2}}{\gamma} Q_{0} \bar{Q}_{0}\right)^{2}
\end{gathered}
$$

In the limit where $M, \bar{M} \ll g v$, the solutions reduce to

$$
\begin{aligned}
& \mathcal{M}_{(0)}^{0}=\sqrt{\gamma(2 \gamma-1)}(M+\bar{M})+\mathcal{O}\left(M^{3}\right) \\
& \mathcal{M}_{(0)}=\frac{\sqrt{2}}{\sqrt{\gamma}} g v+\frac{\gamma^{3 / 2}(M+\bar{M})^{2}}{2 \sqrt{2} g v}+\mathcal{O}\left(M^{4}\right)
\end{aligned}
$$

The dynamics of the second group $\left(Q_{(1)}, \bar{Q}_{(\overline{1})}\right)$ can be split further into three different components. From the couplings to the gauge fields, given in (2.32), it 
appears that the following linear combination

$$
\frac{1}{2 v}\left\{Q_{0}\left(Q_{(1)}-Q_{(1)}^{\dagger}\right)+\bar{Q}_{0}\left(\bar{Q}_{(\overline{1})}-\bar{Q}_{(\overline{1})}^{\dagger}\right)\right\}
$$

corresponds to the would-be-Goldstone bosons that are eaten by the gauge field $A_{\mu(1)}$. Analysis of the spontaneous symmetry breaking mechanism in the potential further reveals that the following linear combination

$$
\frac{1}{2 v}\left\{-\bar{Q}_{0}\left(Q_{(1)}-Q_{(1)}^{\dagger}\right)+Q_{0}\left(\bar{Q}_{(\overline{1})}-\bar{Q}_{(\overline{1})}^{\dagger}\right)\right\}
$$

corresponds to the true massless Goldstone bosons, transforming under the adjoint representation of $S U\left(N_{f}\right)$. The remaining combinations of $Q_{(1)}+Q_{(1)}^{\dagger}$ and $\bar{Q}_{(1)}+\bar{Q}_{(1)}^{\dagger}$ have masses determined by a quadratic equation, given as follows

$$
\begin{aligned}
0= & \left(\mathcal{M}^{2}-2(\gamma-1) M \bar{M}-2(\gamma+1) M^{2}-g^{2} Q_{0}^{2}\right) \times \\
& \times\left(\mathcal{M}^{2}-2(\gamma-1) M \bar{M}-2(\gamma+1) \bar{M}^{2}-g^{2} \bar{Q}_{0}^{2}\right)-g^{4} Q_{0}^{2} \bar{Q}_{0}^{2}
\end{aligned}
$$

In the limit where $M, \bar{M} \ll g v$, the solutions reduce to

$$
\begin{aligned}
& \mathcal{M}_{(1)}^{+}=\sqrt{2} g v+\frac{\gamma(M+\bar{M})^{2}}{2 \sqrt{2} g v}+\mathcal{O}\left(M^{4}\right) \\
& \mathcal{M}_{(1)}^{-}=\sqrt{\gamma}(M+\bar{M})+\mathcal{O}\left(M^{3}\right)
\end{aligned}
$$

The dynamics of the third group $\left(Q_{(2)}, \bar{Q}_{(\overline{2})}\right)$ further splits into two components. From the couplings to the gauge field in (2.32), it appears that the linear combination (and its hermitian conjugate) defined as follows

$$
\frac{1}{\sqrt{2} v}\left(Q_{0} Q_{(2)}+\bar{Q}_{0} \bar{Q}_{(\overline{2})}^{\dagger}\right)
$$

corresponds to the would-be-Goldstone bosons eaten by the gauge field $A_{\mu(2)}$ (and its hermitian conjugate). The remaining linear combination

$$
\frac{1}{\sqrt{2} v}\left(-\bar{Q}_{0} Q_{(2)}+Q_{0} \bar{Q}_{(\overline{2})}^{\dagger}\right)
$$

has mass given by the following exact formula

$$
\mathcal{M}_{(2)}=\sqrt{g^{2} v^{2}+\gamma(M+\bar{M})^{2}}
$$

The results on spin 0 boson masses are summarized in the table 3. The masses of the would-be-Goldstone bosons are referred to by the gauge fields into which they have combined, so the corresponding masses can be found in the table 1 . 


\begin{tabular}{|c|c|c|c|}
\hline Spin 0 & Masses & Multiplicity & $S U\left(N_{f}\right) \times S U\left(N_{c}-N_{f}\right)$ \\
\hline \hline$Q_{(0)}, Q_{(0)}$ & {$\left[A_{\mu(0)}\right], 0, \mathcal{M}_{(0)}^{ \pm}$} & 4 & $1 \otimes 1$ \\
\hline$Q_{(1)}, \bar{Q}_{(\overline{1})}$ & {$\left[A_{\mu(1)}\right], 0, \mathcal{M}_{(1)}^{ \pm}$} & $4\left(N_{f}^{2}-1\right)$ & adjoint $\otimes 1$ \\
\hline$Q_{(2)}, Q_{(\overline{2})}$ & {$\left[A_{\mu(2)}\right], \mathcal{M}_{(2)}$} & $4 N_{f}\left(N_{c}-N_{f}\right)$ & $N_{f}^{*} \otimes\left(N_{c}-N_{f}\right) \oplus$ c.c. \\
\hline
\end{tabular}

Table 3: The spin 0 fields and their masses $\left(N_{f}<N_{c}\right)$

\subsection{Complementarity between confining and Higgs phase}

Let us compare our results with those in ref. [12]. There, the meson fields $T$ are used as fundamental variables in the low energy effective theory. This choice of variables is most appropriate to describe the physics in the confining phase. On the other hand, in the present paper, we have used the fundamental quark fields $Q$ and $\bar{Q}$ instead, which are more appropriate for the description of the physics of the Higgs phase. In nonsupersymmetric gauge theories, with scalar fields in the fundamental representation, it was argued that the Higgs and confining phases are smoothly connected to one another over at least some region of coupling constants [14. Therefore, one should be able to describe the same physics in terms of variables appropriate for either the confining phase or those for Higgs phase.

Thus, we expect that our results should be reproduced using the field variables most suitable for the confining phase, as used in ref. [12]. Naturally, this comparison will only hold in the limit of small supersymmetry breaking mass terms, where the approximations of ref. [12] are valid, and in the limit of the low mass part of the spectrum.

If we use the meson fields $T$ which are appropriate to describe the physics of confining phase, we find the following mass spectrum 7 in the above limit.

1. The spin $1 / 2$ masses for singlet and adjoint are

$$
m_{\mathrm{f}, \text { singlet }}=\frac{\gamma}{k} t^{-(1+\gamma)}, \quad m_{\mathrm{f}, \text { adjoint }}=\frac{1}{k} t^{-(1+\gamma)} .
$$

${ }^{*}$ Here, we have made numerical corrections compared to the original results in ref [12]. However, the conclusions reached in this subsection are not dependent upon these corrections in an essential way. 
2. The spin 0 masses for singlet and adjoint are

$$
m_{\mathrm{s}, \text { singlet }}^{2}=\frac{\gamma(\gamma+1)}{k^{2}} t^{-2(1+\gamma)}, \quad m_{\mathrm{s}, \text { adjoint }}^{2}=\frac{\gamma+1}{k^{2}} t^{-2(1+\gamma)} .
$$

where the vacuum expectation value of $T$ is denoted as $t$. Here we have employed the Ansatz of the Kähler potential for $T$ following ref. [12]

$$
K[\hat{T}]=k \operatorname{tr} \hat{T}^{\dagger} \hat{T} .
$$

If we use the fundamental quark fields $Q, \bar{Q}$ on the other hand, which are appropriate to describe the physics of the Higgs phase, we obtain the following low energy mass spectrum.

1. The spin $1 / 2$ masses for singlet and adjoint are obtained from the first of eqs.(2.37) and (2.40)

$$
m_{\mathrm{f}, \text { singlet }}=(2 \gamma-1) M, \quad m_{\mathrm{f}, \text { adjoint }}=M .
$$

2. The spin 0 masses for singlet and adjoint are obtained from the first of eq.(2.45) and second of eq.(2.49)

$$
m_{\mathrm{s}, \text { singlet }}^{2}=4 \gamma(2 \gamma-1) M^{2}, \quad m_{\mathrm{s}, \text { adjoint }}^{2}=4 \gamma M^{2} .
$$

We see that the qualitative features of the two descriptions are the same, confirming the validity of complementarity between the Higgs and confining phases at a qualitative level. However, we find a discrepancy between the results in the two descriptions upon closer inspection. The mass ratios of the singlet and adjoint particles in the two description are different, both for spin $1 / 2$ as well as spin 0 particles. Although the physical values of the various parameters in the two descriptions cannot be directly related, quantities like the mass ratios should be free of any ambiguities.

The resolution of this discrepancy lies in the following simple remarks. The Kähler potential receives qunatum corrections which cannot be controlled by holomorphy. Therefore the simple Ansatz (2.55) of ref. [12] may not be valid. Moreover, when spontaneous symmetry breaking occurs, and there is a nonvanishing vacuum expectation value for some of the scalar fields, naive dimensional counting of operators has to be modified. In particular, if we are interested in the approximation

\footnotetext{
$\dagger$ In the limit of small supersymmetry breaking parameters, we have $M, \bar{M} \ll g v$.
} 
with at most two derivatives for boson fields and one derivative for fermion fields, then we need to keep all contributions to the Kähler potential in $T$ that only involve the fields $T$ and $T^{\dagger}$. Thus, the Kähler potential used in (2.55) and in ref. [12, is not expected to be sufficient to reproduce completely the low energy behavior of the model, or the low mass part of the spectrum. Instead, we need to include higher order terms in the Kähler potential as well in order to obtain the quadratic terms correctly,

$$
K[T]=k_{1} \operatorname{tr} T^{\dagger} T+k_{2} \operatorname{tr}\left(T^{\dagger} T\right)^{2}+k_{3}\left(\operatorname{tr} T^{\dagger} T\right)^{2}+\cdots
$$

It is easy to see that the parameters $k_{2}, k_{3}$ which are of order $\Lambda^{-2}$, modify the coefficient of the kinetic term as well as the masses of spin $1 / 2$ and 0 particles, in the confining description of ref. [12]. In the limit of large vacuum expectation values, we have a nearly perturbative situation where our assumption for the Kähler potential of the quark superfields is reliable. In this limit, we can find the corresponding Kähler potential for the meson field explicitly as $K[T]=2 \operatorname{tr}\left[\left(T^{\dagger} T\right)^{1 / 2}\right]$ along the D-flat direction.

In principle, similar corrections to the Kähler potential also occur even if we use the fundamental fields $\hat{Q}, \hat{\bar{Q}}$ and $\hat{V}$ which are appropriate to describe the Higgs phase physics. However, there is a well-defined limit, the semi-classical limit, given by $\hbar \rightarrow 0$, where such corrections vanish. In this limit, the vacuum is determined by the minimum of the classical potential, and the masses are just given by the classical fluctuations about this minimum, which is exactly what we used here.

\section{Dynamics for $N_{f}>N_{c}+1$}

In this section, we shall consider the case of $N_{f}>N_{c}+1$. It is most convenient to use the dual variables instead of the original quark and antiquark superfields $\hat{Q}, \hat{\bar{Q}}$ in this case [4]. The dual description for the gauge group $S U\left(N_{c}\right)$ and $N_{f}$ flavors of quarks and antiquarks (with $N_{f}>N_{c}+1$ ) has a gauge group $S U\left(\tilde{N}_{c}\right)$ with $N_{f}$ flavors, where $\tilde{N}_{c}=N_{f}-N_{c}$. The elementary chiral superfields in the dual theory are dual quark $\hat{q}$ and meson $\hat{T}$ superfields,

$$
\hat{q}_{i}^{a} \quad \hat{\bar{q}}_{a}^{i} \quad \hat{T}_{j}^{i} \quad a=1, \cdots, \tilde{N}_{c} ; \quad i, j=1, \cdots N_{f},
$$


which contain scalar fields $q, \bar{q}, T$, and left-handed spinor fields $\psi_{q}, \psi_{\bar{q}}$ and $\psi_{T}$ respectively.

The dual theory has color $S U\left(\tilde{N}_{c}\right)$ gauge invariance, as well as the same global $G_{f}=S U\left(N_{f}\right)_{Q} \times S U\left(N_{f}\right)_{\bar{Q}} \times U(1)_{B} \times U(1)_{R}$ symmetry as the original $S U\left(N_{c}\right)$ theory. The bosonic and left-handed fermionic components transform under these symmetry transformations as

$$
\begin{array}{ccc}
q \rightarrow U_{c} q U_{Q}^{T} & U_{Q} \in S U\left(N_{f}\right)_{Q} \\
\bar{q} \rightarrow U_{\bar{Q}}^{*} \bar{q} U_{c}^{\dagger} & U_{\bar{Q}} \in S U\left(N_{f}\right)_{\bar{Q}} \\
T \rightarrow U_{Q}^{*} T U_{\bar{Q}}^{T} & U_{c} \in S U\left(\tilde{N}_{c}\right)
\end{array}
$$

with baryon number charges $\frac{N_{c}}{\tilde{N}_{c}},-\frac{N_{c}}{\tilde{N}_{c}}$ and 0 respectively, and $R$-charges given by

$$
\begin{array}{cl}
q, \bar{q}: N_{c} / N_{f} & T: 2-2 N_{c} / N_{f} \\
\psi_{q}, \psi_{\bar{q}}: N_{c} / N_{f}-1 & \psi_{T}: 1-2 N_{c} / N_{f}
\end{array}
$$

A further classical axial baryon number $U(1)_{A B}$ symmetry suffers a $S U\left(\tilde{N}_{c}\right)$ color anomaly and is absent at the quantum level.

\subsection{The Effective Potential for Scalar Fields}

Since $\hat{q}, \hat{\bar{q}}$ and $\hat{T}$ are effective fields, their kinetic terms need not have canonical normalizations; in particular, they can receive nonperturbative quantum corrections. Thus, we introduce into the (gauged) Kähler potential for $\hat{q}, \hat{\bar{q}}$ and $\hat{T}$ normalization parameters $k_{q}$ and $k_{T}$ as follows

$$
K[\hat{q}, \hat{\bar{q}}, \hat{T}, \hat{v}]=k_{q} \operatorname{tr}\left(\hat{q}^{\dagger} e^{2 \tilde{g} \hat{v}} \hat{q}+\hat{\bar{q}} e^{-2 \tilde{g} \hat{v}} \hat{\bar{q}}^{\dagger}\right)+k_{T} \operatorname{tr} \hat{T}^{\dagger} \hat{T}
$$

Here, we denote by $\hat{v}$ the $S U\left(\tilde{N}_{c}\right)$ color gauge superfield, and by $\tilde{g}$ the associated coupling constant. (Pure gauge terms will not be exhibited explicitly.) In principle, these normalization parameters are determined by the dynamics of the underlying microscopic theory. Furthermore, it has been pointed out in [4] that a superpotential coupling $q, \bar{q}$ and $T$ should be added as follows

$$
W=\hat{q}_{i}^{a} \hat{T}_{j}^{i} \hat{\bar{q}}_{a}^{j}
$$


We add soft supersymmetry breaking terms to the Lagrangian for the dual quark and meson supermultiplets. Besides gaugino masses, the possible soft terms are

$$
\mathcal{L}_{s b}=-\operatorname{tr}\left(q M_{q}^{2} q^{\dagger}+\bar{q}^{\dagger} M_{\bar{q}}^{2} \bar{q}\right)-M_{T j}^{2 i}{ }^{k}{ }_{l} T^{j}{ }_{i} T^{\dagger l}{ }_{k}-\left(A_{i}{ }^{j}{ }_{k}{ }^{l} \bar{q}^{i}{ }_{a} q^{a}{ }_{j} T^{k}{ }_{l}+\text { h.c. }\right) .
$$

Here $A$ is a cubic coupling, ${ }^{\text {m }}$ and $M_{q}^{2}, M_{\bar{q}}^{2}$ and $M_{T}^{2}$ are mass matrices of $q, \bar{q}$ and $T$ respectively. The $R$-symmetry is broken by the $A$-term in eq.(3.6) and would also be broken by gaugino masses.

For simplicity we shall assume that $R$-symmetry is maintained so that neither $A$-terms nor gaugino masses are present in the Lagrangian. Putting together all $F$ and $D$ terms as well as supersymmetry breaking scalar mass terms, the scalar potential is given by

$$
\begin{aligned}
V(q, \bar{q}, T)= & \frac{1}{k_{T}} \operatorname{tr}\left(q q^{\dagger} \bar{q}^{\dagger} \bar{q}\right)+\frac{1}{k_{q}} \operatorname{tr}\left(q T T^{\dagger} q^{\dagger}+\bar{q}^{\dagger} T^{\dagger} T \bar{q}\right)+\frac{\tilde{g}^{2}}{2}\left(\operatorname{tr} q^{\dagger} \tilde{t}^{a} q-\operatorname{tr} \bar{q} \tilde{t}^{a} \bar{q}^{\dagger}\right)^{2} \\
& +\operatorname{tr}\left(q^{\dagger} M_{q}^{2} q+\bar{q} M_{\bar{q}}^{2} \bar{q}^{\dagger}+T^{\dagger} M_{T}^{2} T\right) .
\end{aligned}
$$

where $\tilde{t}^{a}$ denote generators of $S U\left(\tilde{N}_{c}\right)$.

\subsection{Vacuum Stability}

When the eigenvalues of $M_{q}^{2}, M_{\bar{q}}^{2}$ and $M_{T}^{2}$ can take generic positive or negative values, the scalar potential may be unbounded from below. A necessary condition for which the potential is bounded from below is that $M_{T}^{2}$ be a positive definite matrix. This is because there is no quartic term of $T$.

The D terms vanish when the vacuum expectation values are given by:

$$
\langle 0|q| 0\rangle=\left(\begin{array}{cccc}
q_{1} & & & \\
& \ddots & & 0 \\
& & q_{\tilde{N}_{c}} &
\end{array}\right), \quad\langle 0|\bar{q}| 0\rangle=\left(\begin{array}{ccc}
\bar{q}_{1} & & \\
& \ddots & \\
& & \bar{q}_{\tilde{N}_{c}} \\
& & \\
& & 0
\end{array}\right)
$$

with the combinations $\left|q_{i}\right|^{2}-\left|\bar{q}_{i}\right|^{2}$ independent of $i$.

${ }^{*}$ In general this is a four-index tensor in flavor space, but becomes proportional to $\delta_{i}^{l} \delta_{k}^{j}$ in popular models for soft breaking terms using the supergravity [11]. 
If we set the squark masses to be zero, the space where $\left|q_{i}\right|^{2}$ is independent of $i$ and $\bar{q}=0$ is a subspace of the moduli space of vacua. If we insist on flavor symmetric mass squared matrix and on having a negative eigenvalue, we are forced to have a potential unbounded from below. In fact, in the next subsection, we shall establish more generally that to have a potential bounded from below, we must have

$$
m_{1}^{2}+\cdots+m_{\tilde{N}_{c}}^{2} \geq 0
$$

where $m_{i}^{2}$ are eigenvalues of the matrix $M_{q}^{2}$ or $M_{\bar{q}}^{2}$, and they are set to be $m_{1}^{2} \leq$ $m_{2}^{2} \leq \cdots \leq m_{N_{f}}^{2}$.

Therefore we consider the simplest stable situation, where the $n$ eigenvalues of $M_{q}^{2}$ is negative and same, while all the others are positive or zero. The $n$ should be smaller than $\tilde{N}_{c}$. For simplicity we shall also assume that the soft supersymmetry breaking positive mass squared terms for squarks have a flavor symmetry $S U\left(N_{f}-\right.$ $n)_{Q} \times S U\left(N_{f}\right)_{\bar{Q}}$. As a result, the $N_{f}-n$ positive eigenvalue of $M_{q}^{2}$ are all the same, while the $N_{f}$ eigenvalues of $M_{\bar{q}}^{2}$ are the same $: M_{\bar{q}}^{2}=m_{\bar{q}}^{2} I_{N_{f}}$. We also assume $M_{T}^{2 i}{ }_{j}{ }^{k}{ }_{l}=m_{T}^{2} \delta_{j}^{i} \delta_{l}^{k}$.

\subsection{Determination of VEV}

We begin by making a convenient choice of parameterization for the variables

$q$ and $\bar{q}$. We can always represent the vacuum expectation values of $q, \bar{q}$ and $T$ as follows;

$$
\begin{aligned}
\langle 0|q| 0\rangle & =U_{c(1)}\left(\begin{array}{cc}
q_{(1)} & 0
\end{array}\right) U_{q(1)}^{\dagger}, \\
\langle 0|\bar{q}| 0\rangle & =U_{\bar{q}(1)}\left(\begin{array}{c}
\bar{q}_{(1)} \\
0
\end{array}\right) U_{c(2)}^{\dagger}, \\
\langle 0|T| 0\rangle & =U_{q(2)} T_{(1)} U_{\bar{q}(2)}^{\dagger}
\end{aligned}
$$

where

$$
\begin{gathered}
U_{c(i)} \in S U\left(\tilde{N}_{c}\right), \quad U_{q(i)} \in S U\left(N_{f}\right)_{Q}, \quad U_{\bar{q}(i)} \in S U\left(N_{f}\right)_{\bar{Q}}, \\
q_{(1)}=q_{i} \delta_{i}^{j} \quad \bar{q}_{(1)}=\bar{q}_{i} \delta_{i}^{j} \quad T_{(1)}=T_{i} \delta_{i}^{j}
\end{gathered}
$$


Without loss of generality, the values, $q_{i}, \bar{q}_{i}$ and $T_{i}, i=1, \cdots \tilde{N}_{c}$, can be chosen real and positive.

In this representation, the potential becomes

$$
\begin{aligned}
V(q, \bar{q}, T) & =\frac{1}{k_{T}} \operatorname{tr}\left(q_{(1)}^{2} U_{c} \bar{q}_{(1)}^{2} U_{c}^{\dagger}\right)+\frac{1}{k_{q}} \operatorname{tr}\left[\left(\begin{array}{cc}
q_{(1)}^{2} & 0 \\
0 & 0
\end{array}\right) U_{q}^{\dagger} T_{(1)}^{2} U_{q}+U_{\bar{q}}^{\dagger} T_{(1)}^{2} U_{\bar{q}}\left(\begin{array}{cc}
\bar{q}_{(1)}^{2} & 0 \\
0 & 0
\end{array}\right)\right] \\
& +\frac{\tilde{g}^{2}}{2}\left\{\operatorname{tr}\left(q_{(1)}^{2} \tilde{t}^{a}-U_{c} \bar{q}_{(1)}^{2} U_{c}^{\dagger} \tilde{t}^{a}\right)\right\}^{2} \\
& +\operatorname{tr}\left(U_{q(1)}^{\dagger} M_{q}^{2} U_{q(1)}\left(\begin{array}{cc}
q_{(1)}^{2} & 0 \\
0 & 0
\end{array}\right)+m_{\bar{q}}^{2} \bar{q}_{(1)}^{2}+m_{T}^{2} T_{(1)}^{2}\right)
\end{aligned}
$$

where $U_{c}=U_{c(1)}^{\dagger} U_{c(2)}, U_{q}=U_{q(2)}^{\dagger} U_{q(1)}$, and $U_{\bar{q}}=U_{\bar{q}(2)}^{\dagger} U_{\bar{q}(1)}$.

We would like to find a minima of $V$ as a function of $q_{i}, \bar{q}_{i}, T_{i}$ and the unitary matrices $U$ 's. We notice immediately that the minimum of $V$ is at $T=0$ for fixed $q, \bar{q}$ and unitary matrices. Varying next, at $T=0$, with respect to $U_{c}$ and $U_{q(1)}$, we obtain

$$
\begin{aligned}
{\left[q_{(1)}^{2}, U_{c} \bar{q}_{(1)}^{2} U_{c}^{\dagger}\right] } & =0 \\
{\left[U_{q(1)}^{\dagger} M_{q}^{2} U_{q(1)},\left(\begin{array}{cc}
q_{(1)}^{2} & 0 \\
0 & 0
\end{array}\right)\right] } & =0 .
\end{aligned}
$$

These equations are satisfied only when $U_{c} \bar{q}_{(1)}^{2} U_{c}^{\dagger}$ and $U_{q(1)}^{\dagger} M_{q}^{2} U_{q(1)}$ are diagonal matrices, since $q_{(1)}^{2}$ is assumed to be a generic diagonal matrix. The argument parallels the one given in the discussion of (2.16). (The constraint (3.15) allows the matrix $U_{q(1)}^{\dagger} M_{q}^{2} U_{q(1)}$ to have arbitrary components in the last $N_{f}-\tilde{N}_{c}$ columns and rows. However, we can use the residual flavor symmetry to diagonalize the matrix to obtain the fully diagonal form.)

Since we assume that $n$ eigenvalues of $M_{q}^{2}$ is negative, we obtain the reduced potential

$$
\begin{aligned}
V\left(q_{i}, \bar{q}_{i}\right) & =\frac{1}{k_{T}} \sum_{i=1}^{\tilde{N}_{c}} q_{i}^{2} \bar{q}_{i}^{2}+\frac{\tilde{g}^{2}}{4 \tilde{N}_{c}} \sum_{i<j}^{\tilde{N}_{c}}\left(q_{i}^{2}-\bar{q}_{i}^{2}-q_{j}^{2}+\bar{q}_{j}^{2}\right)^{2} \\
& -m_{q_{1}}^{2} \sum_{i=1}^{n} q_{i}^{2}+m_{q_{2}}^{2} \sum_{i=n+1}^{\tilde{N}_{c}} q_{i}^{2}+m_{\bar{q}}^{2} \sum_{i=1}^{\tilde{N}_{c}} \bar{q}_{i}^{2}
\end{aligned}
$$

up to permutations of $q_{i}$ and $\bar{q}_{i}$.

For arbitrary values of the parameters, this potential is not bounded from below due to the negative sign of the term involving $m_{q_{1}}^{2}$. In order to keep the potential 
bounded from below, the soft-breaking masses must satisfy a condition, which is easily established by considering the special configuration $q_{i}=q, \bar{q}_{i}=0$ :

$$
-n m_{q_{1}}^{2}+\left(\tilde{N}_{c}-n\right) m_{q_{2}}^{2} \geq 0
$$

The extremum conditions for the potential are given by

$$
\begin{aligned}
& q_{i}\left(\frac{1}{k_{T}} \bar{q}_{i}^{2}+\frac{\tilde{g}^{2}}{2 \tilde{N}_{c}}\left\{\left(\tilde{N}_{c}-1\right)\left(q_{i}^{2}-\bar{q}_{i}^{2}\right)-\sum_{k \neq i}\left(q_{k}^{2}-\bar{q}_{k}^{2}\right)\right\}-m_{q_{1}}^{2}\right)=0, \\
& (i=1, \cdots, n) \text {, } \\
& q_{i}\left(\frac{1}{k_{T}} \bar{q}_{i}^{2}+\frac{\tilde{g}^{2}}{2 \tilde{N}_{c}}\left\{\left(\tilde{N}_{c}-1\right)\left(q_{i}^{2}-\bar{q}_{i}^{2}\right)-\sum_{k \neq i}\left(q_{k}^{2}-\bar{q}_{k}^{2}\right)\right\}+m_{q_{2}}^{2}\right)=0 \text {, } \\
& \left(i=n+1, \quad \cdots, \quad \tilde{N}_{c}\right), \\
& \bar{q}_{i}\left(\frac{1}{k_{T}} q_{i}^{2}-\frac{\tilde{g}^{2}}{2 \tilde{N}_{c}}\left\{\left(\tilde{N}_{c}-1\right)\left(q_{i}^{2}-\bar{q}_{i}^{2}\right)-\sum_{k \neq i}\left(q_{k}^{2}-\bar{q}_{k}^{2}\right)\right\}+m_{\bar{q}}^{2}\right)=0 \\
& \left(i=1, \quad \cdots, \quad \tilde{N}_{c}\right) .
\end{aligned}
$$

To solve these equations, we first establish the following facts.

1. For all $i$, we must have $q_{i}^{2} \geq \bar{q}_{i}^{2}$. Indeed, suppose that there exist indices $i_{1}, \cdots, i_{p}$ for which $q_{i_{j}}^{2}<\bar{q}_{i_{j}}^{2}$, while for all remaining indices $i_{k}$ we have $q_{i_{k}}^{2} \geq \bar{q}_{i_{k}}^{2}$. This implies $\bar{q}_{i_{j}} \neq 0$, so that the expression inside the braces in eq. (3.21) has to vanish for $i=i_{1}, \cdots, i_{p}$. Adding those equations, we obtain

$$
\frac{1}{k_{T}} \sum_{j=1}^{p} q_{i_{j}}^{2}-\frac{\tilde{g}^{2}}{2 \tilde{N}_{c}}\left\{\left(\tilde{N}_{c}-p\right) \sum_{j=1}^{p}\left(q_{i_{j}}^{2}-\bar{q}_{i_{j}}^{2}\right)-p \sum_{k \neq i_{1}, \cdots, i_{p}}\left(q_{k}^{2}-\bar{q}_{k}^{2}\right)\right\}+p m_{\bar{q}}^{2}=0 .
$$

Clearly, the left-hand-side is always positive and the equation has no solutions (except for the solution $q=\bar{q}=0$, only when $m_{\bar{q}}^{2}=0$ ).

2. For all $i>n$, either $q_{i}=0$ or $\bar{q}_{i}=0$. Indeed, suppose that both $q_{i} \neq 0$ and $\bar{q}_{i} \neq 0$ for some $i$; then by adding their respective equations in (3.20) and (3.21), one obtains a left-hand-side which is strictly positive, precluding the existence of any solution. Combining this point with the one above in 1., it follows that $\bar{q}_{i}=0$ for all $i>n$.

3. For all $i>n, q_{i}=0$ as well. Indeed, if $q_{1}=\cdots=q_{n}=0$, it follows from (3.20) that $q_{i}$ are equal to zero for all $i$. If $q_{1}^{2}+\cdots+q_{n}^{2} \neq 0$, this is proved similarly 
to 1 applying the stability condition (3.18) to the sum of the expressions in braces in eq. (3.19) and eq. (3.20).

From these facts, we find that the possible solutions of eq. (3.19)-(3.21) are

$$
q_{1}, \cdots, q_{r} \neq 0, \quad \bar{q}_{1}, \cdots, \bar{q}_{s} \neq 0, \quad(n \geq r \geq s \geq 0)
$$

and the others are equal to zero, up to permutations of $q_{i}$ and $\bar{q}_{i}$. They dose not necessarily make minimal.

1. The $r$ should be equal to $n$ at the minimal point. Indeed, for $n \geq i>r$, the second derivative of $q_{i}$ is negative,

$$
\frac{\partial^{2} V}{\partial q_{i}^{2}}=-\frac{\tilde{g}^{2}}{2 \tilde{N}_{c}} \sum_{k \neq i}\left(q_{k}^{2}-\bar{q}_{k}^{2}\right)-m_{q_{1}}^{2}
$$

2. The $s$ should be equal to $n$ or 0 at the minimal point. Indeed, for $n \geq i>s>0$, the second derivative of $\bar{q}_{i}$ is negative on the extremum solution.

After all, we find that there are only two solutions for possible minimum, described as follows.

1. Only $q_{1}, \cdots, q_{n} \neq 0$, while $q_{i}=0, i>n$ and $\bar{q}_{i}=0$ for all $i$. The values of $q_{1}, \cdots, q_{n}$ are the same. We call the common value as $q_{0}$. The value of $q_{0}$ and the potential in this configuration are given by

$$
q_{0}^{2}=\frac{2}{\tilde{g}^{2} \tilde{\gamma}} m_{q_{1}}^{2}, \quad V=-\frac{n}{\tilde{g}^{2} \tilde{\gamma}} m_{q_{1}}^{4}
$$

where

$$
\tilde{\gamma}=\frac{\tilde{N}_{c}-n}{\tilde{N}_{c}}
$$

2. Only $q_{1}, \cdots, q_{n} \neq 0$ and $\bar{q}_{1}, \cdots \bar{q}_{n} \neq 0$, while $q_{i}=\bar{q}_{i}=0, i>n$. The values of $q_{0} \equiv q_{1}=\cdots=q_{n}$ and $\bar{q}_{0} \equiv \bar{q}_{1}=\cdots \bar{q}_{n}$ are then given by

$$
\left(\begin{array}{c}
q_{0}^{2} \\
\bar{q}_{0}^{2}
\end{array}\right)=\frac{1}{\tilde{\gamma} \tilde{g}^{2}-\frac{1}{k_{T}}}\left(\begin{array}{c}
\frac{1}{2} \tilde{\gamma} \tilde{g}^{2} k_{T}\left(m_{q_{1}}^{2}-m_{\bar{q}}^{2}\right)+m_{\bar{q}}^{2} \\
\frac{1}{2} \tilde{\gamma} \tilde{g}^{2} k_{T}\left(m_{q_{1}}^{2}-m_{\bar{q}}^{2}\right)-m_{q_{1}}^{2}
\end{array}\right)
$$


Given the fact that $q_{1}^{2}$ and $\bar{q}_{1}^{2}$ must be positive, this expression yields a solution only when the following condition is satisfied

$$
\frac{1}{2} \tilde{\gamma} \tilde{g}^{2} k_{T}\left(m_{q_{1}}^{2}-m_{\bar{q}}^{2}\right) \geq m_{q_{1}}^{2}
$$

The value of the potential at the stationary point is given by

$$
V=-\frac{n}{4} \frac{\tilde{\gamma} k_{T} \tilde{g}^{2}}{\tilde{\gamma} \tilde{g}^{2}-\frac{1}{k_{T}}}\left(m_{\bar{q}}^{2}-\frac{\frac{1}{2} \tilde{\gamma} \tilde{g}^{2}-\frac{1}{k_{T}}}{\frac{1}{2} \tilde{\gamma} \tilde{g}^{2}} m_{q_{1}}^{2}\right)^{2}-\frac{n}{\tilde{\gamma} \tilde{g}^{2}} m_{q_{1}}^{4}
$$

Therefore, whenever conditions (3.28) is satisfied, solution 2 is the absolute minimum of the potential and describes the true ground state. If condition (3.28) is not satisfied, solution 1 is the absolute minimum.

\subsection{Mass Spectrum}

In our model, the Lagrangian has a global $S U\left(N_{f}-n\right)_{Q} \times S U(n)_{Q} \times U(1)_{Q} \times$ $S U\left(N_{f}\right)_{\bar{Q}} \times U(1)_{B} \times U(1)_{R}$ symmetry. When the coupling $\tilde{g}$ is too weak, the condition (3.28) is not satisfied, solution 1 . is the absolute minimum, and flavor symmetry is not broken. On the other hand, when the gauge coupling is strong, the condition (3.28) is satisfied. Therefore solution 2 is the absolute minimum, and flavor symmetry is spontaneously broken as follows;

$$
\begin{aligned}
& S U\left(N_{f}-n\right)_{Q} \times S U(n)_{Q} \times U(1)_{Q} \times S U\left(N_{f}\right)_{\bar{Q}} \times U(1)_{B} \times U(1)_{R} \\
& \longrightarrow S U\left(N_{f}-n\right)_{Q} \times S U\left(N_{f}-n\right)_{\bar{Q}} \times S U(n)_{V} \times U(1)_{V} \times U(1)_{B^{\prime}} \times U(1)_{R^{\prime}}
\end{aligned}
$$

where $S U(n)_{V}$ is the diagonal subgroup of $S U(n) \subset S U\left(\tilde{N}_{c}\right)$ and $S U(n)_{Q} \times S U(n)_{\bar{Q}}$. The spontaneous breaking of the global symmetry induces spontaneous breaking of color gauge symmetry $S U\left(\tilde{N}_{c}\right) \rightarrow S U\left(\tilde{N}_{c}-n\right)$.

Here we examine the mass spectrum after this spontaneous gauge symmetry breaking, $S U\left(\tilde{N}_{c}\right) \rightarrow S U(n) \times S U\left(\tilde{N}_{c}-n\right)$. To do so, we fix the gauge as follows,

$$
\langle 0|q| 0\rangle=\left(\begin{array}{cc}
q_{0} I_{n} & 0 \\
0 & 0
\end{array}\right) \quad\langle 0|\bar{q}| 0\rangle=\left(\begin{array}{cc}
\bar{q}_{0} I_{n} & 0 \\
0 & 0
\end{array}\right)
$$


and we separate the field variables as follow,

$$
\begin{array}{cc}
q=\left(\begin{array}{cc}
\left(q_{0}+\frac{q_{\langle 0\rangle}}{\sqrt{n}}\right) I_{n}+q_{\langle 1\rangle} & q_{\langle 2\rangle} \\
q_{\langle 3\rangle} & q_{\langle 4\rangle}
\end{array}\right), & \psi_{q}=\left(\begin{array}{cc}
\frac{\psi_{\langle 0\rangle}}{\sqrt{n}} I_{n}+\psi_{\langle 1\rangle} & \psi_{\langle 2\rangle} \\
\psi_{\langle 3\rangle} & \psi_{\langle 4\rangle}
\end{array}\right), \\
\bar{q}=\left(\begin{array}{cc}
\left(\bar{q}_{0}+\frac{\bar{q}_{\langle\overline{0}\rangle}}{\sqrt{n}}\right) I_{n}+\bar{q}_{\langle\overline{1}\rangle} & \bar{q}_{\langle\overline{3}\rangle} \\
\bar{q}_{\langle\overline{2}\rangle} & \bar{q}_{\langle\overline{4}\rangle}
\end{array}\right), & \psi_{\bar{q}}=\left(\begin{array}{cc}
\frac{\psi_{\langle\overline{0}\rangle}}{\sqrt{n}} I_{n}+\psi_{\langle\overline{1}\rangle} & \psi_{\langle\overline{3}\rangle} \\
\psi_{\langle\overline{2}\rangle} & \psi_{\langle\overline{4}\rangle}
\end{array}\right), \\
T=\left(\begin{array}{cc}
\frac{T_{\langle 0\rangle}}{\sqrt{n}} I_{n}+T_{\langle 1\rangle} & T_{\langle 2\rangle} \\
T_{\langle 3\rangle} & T_{\langle 4\rangle}
\end{array}\right), & \psi_{T}=\left(\begin{array}{cc}
\frac{\psi_{T\langle 0\rangle}}{\sqrt{n}} I_{n}+\psi_{T\langle 1\rangle} & \psi_{T\langle 2\rangle} \\
\psi_{T\langle 3\rangle} & \psi_{T\langle 4\rangle}
\end{array}\right) .
\end{array}
$$

The gauge and gaugino fields can be similarly decomposed,

$$
A_{\mu}=\left(\begin{array}{cc}
A_{\mu\langle 1\rangle} & A_{\mu\langle 2\rangle} \\
A_{\mu\langle 2\rangle}^{\dagger} & A_{\mu\langle 3\rangle}
\end{array}\right)+A_{\mu\langle 0\rangle} H_{\langle 0\rangle} \quad \lambda=\left(\begin{array}{cc}
\lambda_{\langle 1\rangle} & \lambda_{\langle 2\rangle} \\
\lambda_{\langle\overline{2}\rangle} & \lambda_{\langle 3\rangle}
\end{array}\right)+\lambda_{\langle 0\rangle} H_{\langle 0\rangle}
$$

where

$$
H_{\langle 0\rangle}=\left(\begin{array}{cc}
C_{1} I_{n} & 0 \\
0 & -C_{2} I_{\tilde{N}_{c}-n}
\end{array}\right)
$$

with

$$
C_{1}=\sqrt{\frac{\tilde{N}_{c}-n}{2 n \tilde{N}_{c}}}, \quad C_{2}=\sqrt{\frac{n}{2 \tilde{N}_{c}\left(\tilde{N}_{c}-n\right)}} .
$$

The fields $q_{\langle 0\rangle}, \bar{q}_{\langle 0\rangle}$, are uniquely defined, provided we insist on the following conditions

$$
\begin{aligned}
& 0=\operatorname{tr} q_{\langle 1\rangle}=\operatorname{tr} \bar{q}_{\langle\overline{1}\rangle}=\operatorname{tr} T_{\langle 1\rangle}=\operatorname{tr} \psi_{\langle 1\rangle}=\operatorname{tr} \psi_{\langle\overline{1}\rangle}=\operatorname{tr} \psi_{T\langle 1\rangle} \\
& 0=\operatorname{tr} A_{\mu\langle 1\rangle}=\operatorname{tr} A_{\mu\langle 3\rangle}=\operatorname{tr} \lambda_{\langle 1\rangle}=\operatorname{tr} \lambda_{\langle 3\rangle}
\end{aligned}
$$

\subsubsection{Spin 1 Masses}

Gauge boson mass terms can be obtained from the $D$-term part of the Lagrangian density, and given by

$$
\begin{aligned}
& -k_{q} \tilde{g}^{2} \operatorname{tr}\left\{\langle 0|q| 0\rangle^{\dagger} A_{\mu} A^{\mu}\langle 0|q| 0\rangle+\langle 0|\bar{q}| 0\rangle A_{\mu} A^{\mu}\langle 0|\bar{q}| 0\rangle^{\dagger}\right\} \\
= & -\frac{1}{2} k_{q} \tilde{g}^{2}\left(q_{0}^{2}+\bar{q}_{0}^{2}\right) \tilde{\gamma} A_{\mu\langle 0\rangle} A_{\langle 0\rangle}^{\mu}-k_{q} \tilde{g}^{2}\left(q_{0}^{2}+\bar{q}_{0}^{2}\right) \operatorname{tr}\left(A_{\mu\langle 1\rangle} A_{\langle 1\rangle}^{\mu}+A_{\mu\langle 2\rangle} A_{\langle 2\rangle}^{\mu \dagger}\right) .
\end{aligned}
$$

It is straightforward to read off the masses of the gauge bosons, and they are summarized in the first table 4 . The would-be-Goldstone bosons that are eaten in order 


\begin{tabular}{|c|c|c|c|}
\hline Spin 1 & Masses $^{2}$ & Multiplicity & $S U\left(\tilde{N}_{c}-n\right)$ \\
\hline \hline$A_{\mu\langle 0\rangle}$ & $k_{q} \tilde{\gamma} \tilde{g}^{2}\left(q_{0}^{2}+\bar{q}_{0}^{2}\right)$ & 1 & 1 \\
\hline$A_{\mu\langle 1\rangle}$ & $k_{q} \tilde{g}^{2}\left(q_{0}^{2}+\bar{q}_{0}^{2}\right)$ & $n^{2}-1$ & 1 \\
\hline$A_{\mu\langle 2\rangle}$ & $\frac{1}{2} k_{q} \tilde{g}^{2}\left(q_{0}^{2}+\bar{q}_{0}^{2}\right)$ & $2 n\left(\tilde{N}_{c}-n\right)$ & $\left(\tilde{N}_{c}-n\right) \oplus\left(\tilde{N}_{c}-n\right)^{*}$ \\
\hline$A_{\mu\langle 3\rangle}$ & 0 & $\left(\tilde{N}_{c}-n\right)^{2}-1$ & adjoint \\
\hline
\end{tabular}

Table 4: The spin 1 fields and their masses in the dual theory

to give these vector bosons mass are identified by inspecting the linear coupling of the scalar particles to the gauge bosons. These couplings are given by

$$
\begin{aligned}
& -k_{q} \tilde{g} \sqrt{2 \tilde{\gamma}} A_{\langle 0\rangle}^{\mu} \partial_{\mu}\left\{\operatorname{Im}\left(q_{0} q_{\langle 0\rangle}+\bar{q}_{0} \bar{q}_{\langle\overline{0}\rangle}^{*}\right)\right\}-2 k_{q} \tilde{g} \operatorname{tr} A_{\langle 1\rangle}^{\mu} \partial_{\mu}\left\{\operatorname{Im}\left(q_{0} q_{\langle 1\rangle}+\bar{q}_{0} \bar{q}_{\langle\overline{1}\rangle}^{\dagger}\right)\right\} \\
& +\left[i k_{q} \tilde{g} \operatorname{tr} A_{\langle 2\rangle}^{\mu} \partial_{\mu}\left(q_{0} q_{\langle 3\rangle}+\bar{q}_{0} \bar{q}_{\langle\overline{3}\rangle}^{\dagger}\right)+\text { h.c. }\right] .
\end{aligned}
$$

The boson field $\operatorname{Im}\left(q_{0} q_{\langle 0\rangle}+\bar{q}_{0} \bar{q}_{\langle\overline{0}\rangle}^{*}\right)$, is eaten by the gauge field $A_{\mu\langle 0\rangle}$, while the boson field, $\operatorname{Im}\left(q_{0} q_{\langle 1\rangle}+\bar{q}_{0} \bar{q}_{\langle\overline{1}\rangle}^{\dagger}\right)$, are eaten by the gauge field, $A_{\mu\langle 1\rangle}$, and the boson field, $\left(q_{0} q_{\langle 3\rangle}+\bar{q}_{0} \bar{q}_{\langle\overline{3}\rangle}^{\dagger}\right)$, (and its complex conjugate) are eaten by the gauge field, $A_{\mu\langle 2\rangle}$.

The properties of the vector bosons are summarized in the table 6 .

\subsubsection{Spin 1/2 Masses}

The fermion mass terms are governed by contributions both from the superpotential (contribution $V_{1}$ ) and from the mixing of the quarks and the gauginos in the $D$-term (contribution $V_{2}$ ) in eq.(2.3). The first contribution, in terms of the irreducible fields of eq. (3.32) is given by

$$
\begin{aligned}
V_{1} & =q_{0}\left\{\psi_{T\langle 0\rangle} \psi_{\langle\overline{0}\rangle}+\operatorname{tr}\left(\psi_{T\langle 1\rangle} \psi_{\langle\overline{1}\rangle}+\psi_{T\langle 2\rangle} \psi_{\langle\overline{2}\rangle}\right)\right\} \\
& +\bar{q}_{0}\left\{\psi_{\langle 0\rangle} \psi_{T\langle 0\rangle}+\operatorname{tr}\left(\psi_{\langle 1\rangle} \psi_{T\langle 1\rangle}+\psi_{\langle 2\rangle} \psi_{T\langle 3\rangle}\right)\right\}+\text { h.c. }
\end{aligned}
$$

Similarly, the contribution $V_{2}$ may be decomposed in terms of the irreducible fields of eq.(3.32), and we obtain

$$
\begin{aligned}
V_{2}= & -i \sqrt{2} \tilde{g} k_{q} \operatorname{tr}\langle 0|q| 0\rangle^{\dagger} \lambda \psi_{q}+i \sqrt{2} \tilde{g} k_{q} \operatorname{tr} \psi_{\bar{q}} \lambda\langle 0|\bar{q}| 0\rangle^{\dagger}+\text { h.c. } \\
= & -i \tilde{g} k_{q} \sqrt{\tilde{\gamma}}\left[q_{0} \lambda_{\langle 0\rangle} \psi_{\langle 0\rangle}-\bar{q}_{0} \psi_{\langle\overline{0}\rangle} \lambda_{\langle 0\rangle}\right] \\
& -i \sqrt{2} \tilde{g} k_{q}\left[q_{0} \operatorname{tr}\left(\lambda_{\langle 1\rangle} \psi_{\langle 1\rangle}+\lambda_{\langle 2\rangle} \psi_{\langle 3\rangle}\right)-\bar{q}_{0} \operatorname{tr}\left(\psi_{\langle\overline{1}\rangle} \lambda_{\langle 1\rangle}+\psi_{\langle\overline{3}\rangle} \lambda_{\langle\overline{2}\rangle}\right)\right]+\text { h.c. }
\end{aligned}
$$


From these mass terms, we can get the mass spectrum, but one must take into account the effective coupling normalizations of the kinetic terms for the spinors. To do so, it is convenient to normalize the fermion fields to canonical expressions for the kinetic terms by the following rescalings

$$
\psi_{q}^{\prime}=\sqrt{k_{q}} \psi_{q}, \quad \psi_{\bar{q}}^{\prime}=\sqrt{k_{q}} \psi_{\bar{q}}, \quad \psi_{T}^{\prime}=\sqrt{k_{T}} \psi_{T}
$$

The gaugino $\lambda$ already has canonical normalization by gauge invariance and need not be rescaled. The fields may be separated into groups transforming under different irreducible representations of the color and flavor groups. Singlets under color symmetry $S U\left(\tilde{N}_{c}-n\right)$ are $\left(\psi_{\langle 2\rangle}, \psi_{T\langle 3\rangle}\right),\left(\psi_{T\langle 2\rangle}, \psi_{\langle\overline{2}\rangle}\right),\left(\lambda_{\langle 0\rangle}, \psi_{\langle 0\rangle}, \psi_{\langle\overline{0}\rangle}, \psi_{T\langle 0\rangle}\right)$, and $\left(\lambda_{\langle 1\rangle}, \psi_{\langle 1\rangle}, \psi_{\langle\overline{1}\rangle}, \psi_{T\langle 1\rangle}\right)$, and have Majorana masses respectively. Fundamental under $S U\left(\tilde{N}_{c}-n\right)$ are $\left(\lambda_{\langle 2\rangle}, \psi_{\langle\overline{3}\rangle}\right)$ and anti-fundamental are $\left(\lambda_{\langle\overline{2}\rangle}, \psi_{\langle 3\rangle}\right)$ which have Dirac masses between each other.

In the set $\left(\lambda_{\langle 0\rangle}, \psi_{\langle 0\rangle}, \psi_{\langle\overline{0}\rangle}, \psi_{T\langle 0\rangle}\right)$, the masses are governed by the quartic equation

$$
0=\mathcal{M}^{4}-\left(\tilde{\gamma} \tilde{g}^{2} k_{q}+\frac{1}{k_{q} k_{T}}\right)\left(q_{0}^{2}+\bar{q}_{0}^{2}\right) \mathcal{M}^{2}+\tilde{\gamma} \frac{1}{k_{T}} \tilde{g}^{2}\left(q_{0}^{2}+\bar{q}_{0}^{2}\right)^{2}
$$

The solutions are

$$
\begin{aligned}
\mathcal{M}_{\langle 0\rangle}^{2} & =k_{q} \tilde{g}^{2} \tilde{\gamma}\left(q_{0}^{2}+\bar{q}_{0}^{2}\right) \\
\mathcal{M}_{T\langle 0\rangle}^{2} & =\frac{1}{k_{q} k_{T}}\left(q_{0}^{2}+\bar{q}_{0}^{2}\right)
\end{aligned}
$$

In the set $\left(\lambda_{\langle 1\rangle}, \psi_{\langle 1\rangle}, \psi_{\langle\overline{1}\rangle}, \psi_{T\langle 1\rangle}\right)$, the masses are governed by the quartic equation

$$
0=\mathcal{M}^{4}-\left(2 \tilde{g}^{2} k_{q}+\frac{1}{k_{q} k_{T}}\right)\left(q_{0}^{2}+\bar{q}_{0}^{2}\right) \mathcal{M}^{2}+2 \frac{1}{k_{T}} \tilde{g}^{2}\left(q_{0}^{2}+\bar{q}_{0}^{2}\right)^{2}
$$

The solutions are

$$
\begin{aligned}
\mathcal{M}_{\langle 1\rangle}^{2} & =2 k_{q} \tilde{g}^{2}\left(q_{0}^{2}+\bar{q}_{0}^{2}\right) \\
\mathcal{M}_{T\langle 1\rangle}^{2} & =\frac{1}{k_{q} k_{T}}\left(q_{0}^{2}+\bar{q}_{0}^{2}\right)
\end{aligned}
$$

A summary of all spin $1 / 2$ fields and masses is given in the table 5 . 


\begin{tabular}{|c|c|c|c|}
\hline Spin $1 / 2$ & Masses $^{2}$ & Multiplicity & $S U\left(\tilde{N}_{c}-n\right)$ \\
\hline \hline$\psi_{\langle 2\rangle}, \psi_{T\langle 3\rangle}$ & $\frac{1}{k_{q} k_{T}} \bar{q}_{0}^{2}$ & $2 n\left(N_{f}-n\right)$ & 1 \\
\hline$\psi_{T\langle 2\rangle}, \psi_{\langle\overline{2}\rangle}$ & $\frac{1}{k_{q} k_{T}} q_{0}^{2}$ & $2 n\left(N_{f}-n\right)$ & 1 \\
\hline$\lambda_{\langle 0\rangle}, \psi_{T\langle 0\rangle}$, & $\mathcal{M}_{\langle 0\rangle}^{2}$ & 4 & 1 \\
$\psi_{\langle 0\rangle}, \psi_{\langle\overline{0}\rangle}$ & $\mathcal{M}_{T\langle 0\rangle}^{2}$ & & \\
\hline$\lambda_{\langle 1\rangle}, \psi_{T\langle 1\rangle}$, & $\mathcal{M}_{\langle 1\rangle}^{2}$ & $4\left(n^{2}-1\right)$ & 1 \\
$\psi_{\langle 1\rangle}, \psi_{\langle\overline{1}\rangle}$ & $\mathcal{M}_{T\langle 1\rangle}^{2}$ & & \\
\hline$\left(\lambda_{\langle 2\rangle}, \psi_{\langle\overline{3}\rangle}\right)$ & & $2 n\left(\tilde{N}_{c}-n\right)$ & $\tilde{N}_{c}-n$ \\
$\left(\lambda_{\langle\overline{2}\rangle}, \psi_{\langle 3\rangle}\right)$ & $2 \tilde{g}^{2} k_{q} q_{1}^{2}, 2 \tilde{g}^{2} k_{q} \bar{q}_{1}^{2}$ & & \\
& & $2 n\left(\tilde{N}_{c}-n\right)$ & $\left(\tilde{N}_{c}-n\right)^{*}$ \\
\hline
\end{tabular}

Table 5: The spin 1/2 fields and their masses in the dual theory

\subsubsection{Spin 0 Masses}

We give the mass terms of spin 0 masses in terms of the irreducible fields of eq.(3.32).

In the case of weak coupling, the mass terms are as follows,

$$
\begin{aligned}
-\mathcal{L}_{\text {mass }} & =m_{q_{1}}^{2}\left(q_{\langle 0\rangle}+q_{\langle 0\rangle}^{*}\right)^{2}+\frac{1}{\tilde{\gamma}} m_{q_{1}}^{2} \operatorname{tr}\left(q_{\langle 1\rangle}+q_{\langle 1\rangle}^{\dagger}\right)^{2} \\
& +\left(m_{q_{1}}^{2}+m_{q_{2}}^{2}\right) \operatorname{tr} q_{\langle 2\rangle} q_{\langle 2\rangle}^{\dagger}+\left(m_{q_{2}}^{2}-\frac{n}{\tilde{N}_{c}-n} m_{q_{1}}^{2}\right) \operatorname{tr} q_{\langle 4\rangle} q_{\langle 4\rangle}^{\dagger} \\
& +\left\{\left(\frac{2}{\tilde{g}^{2} \tilde{\gamma}} \frac{1}{k_{T}}-1\right) m_{q_{1}}^{2}+m_{\bar{q}}^{2}\right\}\left(\bar{q}_{\langle\overline{0}\rangle}^{*} \bar{q}_{\langle\overline{0}\rangle}+\operatorname{tr} \bar{q}_{\langle\overline{1}\rangle}^{\dagger} \bar{q}_{\langle\overline{1}\rangle}+\operatorname{tr} \bar{q}_{\langle\overline{2}\rangle}^{\dagger} \bar{q}_{\langle\overline{2}\rangle}\right) \\
& +\left(m_{\bar{q}}^{2}+\frac{n}{\tilde{N}_{c}-n} m_{q_{1}}^{2}\right) \operatorname{tr}\left(\bar{q}_{\langle\overline{3}\rangle}^{\dagger} \bar{q}_{\langle\overline{3}\rangle}+\bar{q}_{\langle\overline{4}\rangle}^{\dagger} \bar{q}_{\langle\overline{4}\rangle}\right) \\
& +\left(\frac{1}{k_{q}} \frac{2}{\tilde{g}^{2} \tilde{\gamma}} m_{q_{1}}^{2}+m_{T}^{2}\right)\left(T_{\langle 0\rangle} T_{\langle 0\rangle}^{*}+\operatorname{tr} T_{\langle 1\rangle} T_{\langle 1\rangle}^{\dagger}+\operatorname{tr} T_{\langle 2\rangle} T_{\langle 2\rangle}^{\dagger}\right) \\
& +m_{T}^{2} \operatorname{tr}\left(T_{\langle 3\rangle} T_{\langle 3\rangle}^{\dagger}+T_{\langle 4\rangle} T_{\langle 4\rangle}^{\dagger}\right)
\end{aligned}
$$

The bosons, $\operatorname{Im} q_{\langle 0\rangle}, q_{\langle 1\rangle}-q_{\langle 1\rangle}^{\dagger}, q_{\langle 3\rangle}$ and $q_{\langle 3\rangle}^{\dagger}$ will be eaten by gauge bosons. There is no genuine Nambu-Goldstone boson in this case.

In the case of strong coupling, the mass spectrum is as follows;

$$
\begin{aligned}
-\mathcal{L}_{\text {mass }} & =\frac{1}{4} \tilde{\gamma} \tilde{g}^{2} q_{0}^{2}\left(q_{\langle 0\rangle}+q_{\langle 0\rangle}^{*}\right)^{2}+\frac{1}{4} \tilde{\gamma} \tilde{g}^{2} \bar{q}_{0}^{2}\left(\bar{q}_{\langle\overline{0}\rangle}+\bar{q}_{\langle\overline{0}\rangle}^{*}\right)^{2} \\
& -\left(\frac{1}{2} \tilde{\gamma} \tilde{g}^{2}-\frac{1}{k_{T}}\right) q_{0} \bar{q}_{0}\left(q_{\langle 0\rangle}+q_{\langle 0\rangle}^{*}\right)\left(\bar{q}_{\langle\overline{0}\rangle}+\bar{q}_{\langle\overline{0}\rangle}^{*}\right)
\end{aligned}
$$




$$
\begin{aligned}
& +\frac{1}{4} \tilde{g}^{2} q_{0}^{2} \operatorname{tr}\left(q_{\langle 1\rangle}+q_{\langle 1\rangle}^{\dagger}\right)^{2}+\frac{1}{4} \tilde{g}^{2} \bar{q}_{0}^{2}\left(\bar{q}_{\langle\overline{1}\rangle}+\bar{q}_{\langle\overline{1}\rangle}^{\dagger}\right)^{2} \\
& -\left(\frac{1}{2} \tilde{g}^{2}-\frac{1}{k_{T}}\right) q_{0} \bar{q}_{0} \operatorname{tr}\left(q_{\langle 1\rangle}+q_{\langle 1\rangle}^{\dagger}\right)\left(\bar{q}_{\langle\overline{1}\rangle}+\bar{q}_{\langle\overline{1}\rangle}^{\dagger}\right) \\
& +\left(m_{q_{1}}^{2}+m_{q_{2}}^{2}\right) \operatorname{tr} q_{\langle 2\rangle} q_{\langle 2\rangle}^{\dagger}+\left(\frac{1}{2} \tilde{g}^{2} k_{T}-1\right)\left(m_{q_{1}}^{2}-m_{\bar{q}}^{2}\right)\left|\frac{\bar{q}_{0} q_{\langle 3\rangle}-q_{0} \bar{q}_{\langle\overline{3}\rangle}^{\dagger}}{\sqrt{q_{0}^{2}+\bar{q}_{0}^{2}}}\right|^{2} \\
& +\left(m_{q_{2}}^{2}-\frac{n}{\tilde{N}_{c}-n} \frac{\frac{1}{2} \tilde{\gamma} \tilde{g}^{2}}{\tilde{\gamma} \tilde{g}^{2}-\frac{1}{k_{T}}}\left(m_{q_{1}}^{2}+m_{\bar{q}}^{2}\right)\right) \operatorname{tr} q_{\langle 4\rangle} q_{\langle 4\rangle}^{\dagger} \\
& +\left(m_{\bar{q}}^{2}+\frac{n}{\tilde{N}_{c}-n} \frac{\frac{1}{2} \tilde{\gamma} \tilde{g}^{2}}{\tilde{\gamma} \tilde{g}^{2}-\frac{1}{k_{T}}}\left(m_{q_{1}}^{2}+m_{\bar{q}}^{2}\right)\right) \operatorname{tr} \bar{q}_{\langle\overline{4}\rangle}^{\dagger} \bar{q}_{\langle\overline{4}\rangle} \\
& +\left\{\frac{k_{T}}{k_{q}}\left(m_{q_{1}}^{2}-m_{\bar{q}}^{2}\right)+m_{T}^{2}\right\}\left(T_{\langle 0\rangle} T_{\langle 0\rangle}^{*}+\operatorname{tr} T_{\langle 1\rangle} T_{\langle 1\rangle}^{\dagger}\right) \\
& +\left(\frac{1}{k_{q}} q_{0}^{2}+m_{T}^{2}\right) \operatorname{tr} T_{\langle 2\rangle} T_{\langle 2\rangle}^{\dagger}+\left(\frac{1}{k_{q}} \bar{q}_{0}^{2}+m_{T}^{2}\right) \operatorname{tr} T_{\langle 3\rangle}^{\dagger} T_{\langle 3\rangle}+m_{T}^{2} \operatorname{tr} T_{\langle 4\rangle}^{\dagger} T_{\langle 4\rangle}
\end{aligned}
$$

The bosons, $\operatorname{Re} q_{\langle 1\rangle}$ and $\operatorname{Re} \bar{q}_{\langle\overline{1}\rangle}$, will mix. The mass eigenvalues are governed by the equation

$$
0=\left(\mathcal{M}^{2}\right)^{2}-\frac{1}{4 k_{q}} \tilde{\gamma} \tilde{g}^{2}\left(q_{0}^{2}+\bar{q}_{0}^{2}\right) \mathcal{M}^{2}+\frac{1}{4 k_{T} k_{q}}\left(\tilde{\gamma} \tilde{g}^{2}-\frac{1}{k_{T}}\right) q_{0}^{2} \bar{q}_{0}
$$

The solutions in the limit where $\tilde{g}^{2} \gg 1$ are

$$
\mathcal{M}^{2}=\tilde{\gamma} \tilde{g}^{2} \frac{k_{T}}{k_{q}}\left(m_{q_{1}}^{2}-m_{\bar{q}}^{2}\right), \quad m_{q_{1}}^{2}-m_{\bar{q}}^{2} .
$$

As shown in previous section, the bosons, $\operatorname{Im}\left(q_{0} q_{\langle 0\rangle}+\bar{q}_{0} \bar{q}_{\langle\overline{0}\rangle}^{*}\right), \operatorname{Im}\left(q_{0} q_{\langle 1\rangle}+\bar{q}_{0} \bar{q}_{\langle\overline{1}\rangle}^{\dagger}\right)$, $\left(q_{0} q_{\langle 3\rangle}+\bar{q}_{0} \bar{q}_{\langle\overline{3}\rangle}^{\dagger}\right)$, and its complex conjugates are would-be-Goldstone bosons which are eaten by gauge bosons. The bosons, $\operatorname{Im}\left(\bar{q}_{0} q_{\langle 0\rangle}-q_{0} \bar{q}_{\langle\overline{0}\rangle}^{*}\right), \operatorname{Im}\left(\bar{q}_{0} q_{\langle 1\rangle}-q_{0} \bar{q}_{\langle\overline{1}\rangle}^{\dagger}\right)$, $\bar{q}_{\langle\overline{2}\rangle}$ and $\bar{q}_{\langle\overline{2}\rangle}^{\dagger}$ are genuine Nambu-Goldstone bosons corresponding to $S U\left(N_{f}\right) \rightarrow$ $S U\left(N_{f}-n\right)_{\bar{Q}} \times S U(n)_{V}$.

\section{Discussion on the validity of our analysis}

It has been found that the classical moduli space is modified quantum mechanically for cases $N_{f}=N_{c}$ [3], [4]. For $N_{f} \geq N_{c}$, we can define baryon fields

$$
B^{i_{1}, \cdots, i_{N_{c}}}=\epsilon_{a_{1}, \cdots, a_{N_{c}}} Q_{a_{1}}^{i_{1}} \cdots Q_{a_{N_{c}}}^{i_{N_{c}}} .
$$


In terms of the baryon and antibaryon field $B$ and $\bar{B}$ and meson field $T$ in eq.(2.2), there is a constraint classically for $N_{f}=N_{c}$ which is modified as

$$
\operatorname{det} T-B \bar{B}=0 \rightarrow \operatorname{det} T-B \bar{B}=\Lambda^{2 N_{c}}
$$

In such a situation, the quantum fluctuations are important and these meson and baryon fields cannot be treated semi-classically as a product of elementary quark fields. In particular, we cannot regard the vacuum expectation value of the meson and baryon fields to be a product of those of the elementary squark fields. Therefore even the analysis of vacuum configuration requires more than a semi-classical treatment which is used in our paper. This phenomenon clearly suggests that the semi-classical treatment of these meson and baryon fields as a product of elementary quark fields is not accurate.

For $N_{f}=N_{c}+1$, the classical moduli space is not modified [3], [4].

$$
B_{i} T^{i}{ }_{j} \bar{B}^{j}-\operatorname{det} T=0
$$

Therefore we can use the classical description for vacuum expectation values. On the other hand, the nonperturbative potential is given in terms of this combination of scalar fields

$$
W=B_{i} T_{j}^{i} \bar{B}^{j}-\operatorname{det} T
$$

If the scalar meson and baryon fields are just the simple product of scalar quark fields, the relation (4.3) should hold for fields themselves. Therefore if we take fundamental fields $Q$ and $\bar{Q}$ as dynamical variables and regard the composite fields $T, B$, and $\bar{B}$ as merely the naive products of them, the superpotential vanishes identically. Hence the superpotential (4.4) implies that we cannot regard the meson and baryon fields to be the products of quark fields, and the quantum fluctuations are important for these composite fields.

At the moment we have no good means of analyzing the case where quantum mechanical effects are really important such as $N_{f}=N_{c}$ and $N_{f}=N_{c}+1$. Our analysis should be reliable away from these situations $\left(N_{f} \ll N_{c}\right.$ or $\left.N_{f} \gg N_{c}\right)$ and hopefully carries a qualitatively correct result even for situations where such quantum effects are important.

Let us finally reconsider the region of validity of our analysis. The most reliable parameter region is clearly the case of small soft mass terms (compared to the gauge interaction scale $\Lambda$ ). In order to avoid the vacuum instability in the limit of vanishing soft mass terms, we can add a small supersymmetric mass. If we do not have 
the soft mass terms and have only a generic supersymmetric mass together with the nonperturbative superpotential, we obtain a stable supersymmetric vacuum with vacuum expectation values for squarks which break gauge as well as flavor symmetries. We can introduce the soft mass terms to this system and repeat the analysis almost identical to the one in this paper. Then we find that the results smoothly connect to those of the supersymmetric vacuum in the limit of vanishing soft mass terms. The region near this situation is most reliable and is nearly perturbative. As we increase the value of soft mass terms, we may encounter modifications of the nonperturbative effects due to the soft mass terms. Assuming such modifications are small, we can explore an interesting situation where the gauge (and flavor) symmetries are broken mainly by the negative soft mass squared terms and calculate the nonperturbative corrections for vacuum expectation values and masses due to the (mildly strong) gauge interactions. This possibility is a genuinely new interesting case, although it clearly involves more assumptions. This assumption is partly supported by our analysis of soft breaking terms using the spurion in sect. 2.1. By the similar token, our analysis for the case of $N_{f}>N_{c}$ is accurate when we have small soft mass squared terms for dual quarks. However, the relation between dual quark field and the original fundamental quark field is nonperturbative and complicated. Therefore the relation between the soft mass terms of the original squark field and those of the dual quark fields may not be simple. This ambiguity is reflected in the fact that we have the unknown coefficient for the Kähler potential of the kinetic term for dual quarks $k_{q}$ and for mesons $k_{T}$ in eq.(3.4). In this respect, our analysis for the case $N_{f}>N_{c}$ involves more assumptions than that of $N_{f}<N_{c}$ case.

We acknowledge the hospitality of Institute for Theoretical Physics in Santa Barbara for providing the stimulating atmosphere in which most of this research was carried out. Also, Y.M and N.S. thank the Department of Physics at UCLA for their hospitality. This work is partially supported by the US-Japan collaborative program, by National Science Foundation Grant PHY-92-18990 and by Grant-inAid for Scientific Research (Y.M.) and (No.05640334 for N.S.) from the Japanese Ministry of Education, Science and Culture.

\section{References}


[1] N. Seiberg and E. Witten, Nucl. Phys. B426 (1994) 19, ibid. Nucl. Phys. B431 (1994) 484; C. Vafa and E. Witten, Nucl. Phys. B431 (1994) 3.

[2] G. Veneziano and S. Yankielowicz, Phys. Lett. 113 (1982) 231; T. Taylor, G. Veneziano and S. Yankielowicz, Nucl. Phys. B218 (1983) 493.

[3] J. Affleck, M. Dine and N. Seiberg, Phys. Rev. Lett. 51 (1983) 1026, ibid. Nucl. Phys. B241 (1984) 493.

[4] N. Seiberg, Phys. Rev. D49 (1994) 6857, ibid. Nucl. Phys. B435 (1995) 129, hep-th.9408013.

[5] K. Intriligator, R. Leigh, and N. Seiberg, Phys. Rev. D50 (1994) 1092; K. Intriligator and N. Seiberg, Nucl. Phys. B431 (1994) 551, Nucl. Phys. B447 (1995) 125, hep-th.9509066; M. Douglas and S. Shenker, Nucl. Phys. B447 (1995) 271; S. Elitzur, A. Forge, A. Giveon and E. Rabinovici, Phys. Lett. B353 (1995) 79.

[6] P. Fayet and J. Iliopoulos, Phys. Lett. B51 (1974) 461.

[7] L. O’Raifeartaigh, Nucl. Phys. B96 (1975) 331.

[8] L. Girardello and M. Grisaru, Nucl. Phys. 194B (1982) 65; K. Harada and N. Sakai, Prog. Theor. Phys. 67 (1982) 1877.

[9] N. Sakai, Zeit. f. Phys. 11 (1981) 153.

[10] S. Dimopoulos and H. Georgi, Nucl. Phys. B193 (1981) 150.

[11] For a review see for instance, H.P. Nilles, Phys. Rep. C110 (1984) 1; P. Nath, R. Arnowitt, and A. Chamseddine, Applied $N=1$ Supergravity, the ICTP Series in Theoretical Physics, Vol. I (World scientific) 1984; H. Haber and G. Kane, Phys. Rep. C117 (1985) 75.

[12] O. Aharony, M. Peskin, J. Sonnenschein, and S. Yankielowicz, Phys. Rev. D52 (1995) 6157, hep-th.9509165.

[13] J. Preskill and S. Weinberg, Phys. Rev. D24 (1981) 1059; S. Dimopoulos and J. Preskill, Nucl. Phys. B199 (1982) 206. 
[14] E. Fradkin and S. Shenker, Phys. Rev. D19 (1979) 3682; T. Banks and E. Rabinovici, Nucl. Phys. B160 (1979) 349; S. Dimopoulos, S. Raby, and L. Susskind, Nucl. Phys. B173 (1980) 208.

[15] N. Evans, S.D.H.Hsu, and M. Schwetz, Phys. Lett. B355 (1995) 475; N. Evans, S.D.H.Hsu, M. Schwetz, and S. B. Selipsky, Nucl. Phys. B456 (1995) 205; 